\title{
REQUISITOS MÍNIMOS DE DETALLADO DÚCTIL EN MARCOS DE CONCRETO REFORZADO PROTEGIDOS CON DISIPADORES HISTERÉTICOS DE ENERGÍA
}

\author{
Horacio de Jesús Nangullasmú Hernández ${ }^{(1)}$ y Arturo Tena Colunga ${ }^{(2)}$
}

\begin{abstract}
RESUMEN
En este trabajo los autores presentan los resultados de un estudio paramétrico, efectuados mediante análisis estáticos no lineales (pushover), de marcos de concreto reforzado con dispositivos de disipación de energía por histéresis montados en contraventeo chevrón, donde se evalúa la eficiencia estructural global de proporcionar requisitos de detallado dúctil a los elementos de concreto, adicionales a los requerimientos de diseño para marcos no dúctiles de los reglamentos de México. Se diseñaron marcos cuyas elevaciones oscilaron entre 5 y 25 niveles, utilizando diferentes balances de rigidez entre el sistema contraviento-dispositivo histerético y el marco resistente a momento $(\alpha)$, así como de la rigidez elástica entre el disipador histerético y los contravientos de soporte $(\beta)$. Se consideró una pendiente posterior a la fluencia del $5 \%$ para los disipadores de energía y un ángulo de inclinación de contravientos con respecto al eje horizontal $\left(\theta=45^{\circ}\right)$. A partir de los resultados obtenidos, se ampliaron las posibles combinaciones óptimas de rigidez de este tipo de estructuras con respecto a las obtenidas previamente en modelos con detallado no dúctil en los elementos de concreto. Se discute el impacto en la capacidad de deformación del sistema cuando se usan o no requisitos mínimos de detallado dúctil en este tipo de configuración estructural, y se obtienen valores para los factores de comportamiento sísmico $(Q)$ y de sobrerresistencia $(R)$.
\end{abstract}

Palabras clave: disipadores de energía; marcos de concreto reforzado; balances de rigideces; ductilidad; sobrerresistencia

\section{MINIMUM DUCTILE CONFINEMENT REQUIREMENTS IN REINFORCED CONCRETE FRAMES PROTECTED WITH HYSTERETIC ENERGY DISSIPATION DEVICES}

\begin{abstract}
In this paper the authors summarize results obtained from pushover analyses for reinforced concrete intermediate moment-resisting frames (RC-IMRFs) with hysteretic energy dissipation devices (HEDDs), mounted in chevron steel bracing. The global structural efficiency was evaluated for the RC-IMRFs designed with minimum ductile confinement requirements as an additional design request specified in the reinforced concrete guidelines of Mexican codes. Moment-resisting frames ranged from 5 to 25 stories and were designed using diverse stiffness ratios between frame system

\footnotetext{
Artículo recibido el 29 de enero de 2016 y aprobado para su publicación el 5 de noviembre de 2016. Se aceptarán comentarios y/o discusiones hasta cinco meses después de su publicación.

(1) Posgrado de Ingeniería Estructural, Universidad Autónoma Metropolitana Azcapotzalco, Av. San Pablo \#180, 02200, México, DF, e-mail: hnangu@ hotmail.com

(2) Profesor, Departamento de Materiales, Universidad Autónoma Metropolitana Azcapotzalco, Av. San Pablo 180, Col. Reynosa Tamaulipas, 02200 México, DF, e-mail: atc@ correo.azc.uam.mx
} 
and the whole structure $(\alpha)$, as well as stiffness ratios between the HEDD and the supporting brace $(\beta)$. A post to pre yielding stiffness ratio $\left(\mathrm{k}_{2} / \mathrm{k}_{\mathrm{EL}}\right)$ of $5 \%$ for the hysteretic devices was also considered. An angle of inclination of the chevron braces with respect to the horizontal axis $\theta=45^{\circ}$ was taken into account. The structural behavior of RC-IMRFs with these extra requirements is much improved with respect of the typical detailing established in Mexican codes for RC-IMRFs. Updated seismic global design parameters related to global ductility $(Q)$ and overstrength $(R)$ that such structures could develop were assessed. Finally, comments related to the use of minimum ductile confinement requirements are exposed with the main objective to induce a "practical" design procedure for this kind of structures.

Keywords: energy dissipation devices; stiffness balances; reinforced concrete frames; ductility; overstrength

\section{INTRODUCCIÓN}

Los reglamentos de construcción en México se enfocan en preservar la vida de sus habitantes con base en la disipación de la energía sísmica por medio del daño controlado de sus componentes estructurales principales (asociado a pérdidas de rigidez y resistencia de los elementos primarios). En la mayoría de los casos, esta filosofía de diseño tiene como consecuencia la suspensión de actividades, mientras duran los trabajos de reparación necesarios en una estructura dada, en los casos que esto sea viable. Una alternativa que resultó atractiva para los ingenieros connacionales, después del sismo suscitado en septiembre de 1985, fue el control de la respuesta sísmica debido a que, además de proteger la vida de las personas, pretende garantizar la operatividad y rentabilidad de cualquier estructura después de que ocurra un sismo de magnitud considerable.

Existen diversas investigaciones acerca de las aplicaciones de disipadores por histéresis del material y muchas más acerca de los sistemas de control de la respuesta sísmica, los cuáles reportan el uso de éstos últimos desde hace más de 100 años, por ejemplo, cuando en Japón John Milne aisló sísmicamente una casa de madera con apoyos esféricos deslizantes (Housner et al. 1997). Durante la última década los investigadores han profundizado sus esfuerzos en diversos proyectos experimentales y analíticos, donde exploran la eficiencia de nuevas configuraciones geométricas de disipadores de energía (Mualla y Belev 2002, Shih y Sung 2005, Chan y Albermani 2008, Ghabraie et al. 2010), valoran el uso de nuevos materiales para la creación de tales dispositivos (Sahoo y Rai 2010, Rai et al. 2013) o evalúan nuevas configuraciones estructurales para la colocación de los disipadores, con la finalidad de crear uno que se desempeñe de manera eficiente, pero a menor costo que los más conocidos y ampliamente estudiados.

Aunque desde hace muchos años atrás se ha abordado la problemática de encontrar el lugar óptimo para la colocación de dispositivos disipadores de energía que sean capaces de mitigar los efectos traslacionales y/o torsionales de los sismos en las estructuras (por ejemplo, Wu et al. 1997), existen una serie de investigaciones experimentales, desarrollados en la última década, que presentan propuestas de nuevos sistemas de disipación, que no necesariamente se encuentran colocados sobre sistemas de contraventeo chevrón o concéntrico. Tal es el caso de las investigaciones realizadas por Oh et al. (2009), Castiglioni et al. (2012) y Calado et al. (2013).

Dentro de los investigaciones analíticas desarrolladas con disipadores de energía por histéresis de material, existen aquéllas que se han enfocado en proponer nuevas metodologías de diseño, algunas tomando como fundamento códigos de diseño de sus respectivos países (Lin et al. 2003, Mahmoudi y Abdi 2012, Karavasilis et al. 2012) y algunas otras incorporando el concepto de amortiguamiento viscoso equivalente (por ejemplo, Hanson 1993, Foti et al. 1998, Hanson y Soong 2001). Por otra parte, otros se 
limitan a comparar resultados de métodos analíticos con los obtenidos de ensayes experimentales para una posible predicción del comportamiento estructural global (Tehranizadeh 2001, Zahrai y Moslehi 2013). Los trabajos de Jara et al. (2007), de Vargas y Bruneau (2009), de Jara (2009), de Tena y Nangullasmú (2013) y Tena-Colunga y Nangullasmú-Hernández (2015) complementan de manera adecuada un amplio panorama de estructuras con disipadores de energía evaluados con la hipótesis de balances de rigidez entre sus sistemas. Estas investigaciones varían desde sistemas de un grado de libertad con comportamiento elastoplástico, hasta sistemas de múltiples grados de libertad con pendientes posteriores a fluencia. Los análisis realizados por los investigadores antes mencionados son del tipo estático y dinámico no lineal desplantados en terreno firme y terreno blando. Además de estas diferencias de parámetros, existen otras variaciones tomadas en cuenta para la obtención de recomendaciones para la aplicación de disipadores en estructuras sismorresistentes. Aunque no se pueden comparar de manera directa los resultados obtenidos, el comportamiento observado del universo de modelos evaluados es bastante similar. Por ejemplo, se observó que la relación de rigidez entre el contraviento y el disipador influye directamente en la ductilidad última desarrollada por los disipadores. Además, la relación de rigidez entre el sistema del marco y del contraviento-disipador debe incrementarse conforme los modelos analizados aumentan en altura.

La idea de usar balances de rigidez como propuesta de metodología de diseño se ha usado por diversos investigadores, y quizás fue iniciado a nivel mundial por Ciampi et al. (1992 y 1995) para disipadores histeréticos. En este sentido, uno de los primeros trabajos desarrollados en México con este criterio para sistemas estructurales con disipadores de energía fue el presentado por Esteva y Veras (1998). Ellos resaltan que para sistemas mixtos (marcos resistentes a momento y disipación de energía), describir las propiedades de sus elementos estructurales con base exclusivamente en su amortiguamiento efectivo es incompleto, ya que además del incremento de esta propiedad, el sistema completo sufre cambios en su rigidez y resistencia (Esteva y Veras 1998). Esteva y Veras destacan la importancia de identificar los diferentes niveles de ductilidad que pueden llegar a desarrollar los múltiples elementos de dicho sistema estructural mixto, mediante una ductilidad equivalente y el empleo de un conjunto de espectros de diseño. No obstante, los resultados del estudio paramétrico presentado están orientados a una filosofía de diseño basada en confiabilidad y en índices de daño. Los dos principales parámetros que evalúan son denotados por las letras " $\alpha$ " y " $\beta$ ", que representan cocientes de balances de rigidez y de resistencia entre el disipador y el marco respectivamente.

Con el propósito de fomentar el uso de estructuras con dispositivos de control pasivo de la respuesta sísmica adaptando procedimientos de diseño comúnmente utilizados para diseñar sistemas estructurales convencionales, se presenta un estudio paramétrico de marcos no dúctiles de concreto con disipadores por histéresis de energía montados en contraventeo chevrón, proponiendo la adición de confinamiento dúctil a los elementos de concreto, con el objetivo de mejorar el desempeño estructural global evaluados a partir de los mapeos de fluencia principalmente. Se parte de una metodología de diseño acorde a balances de rigidez entre el sistema de marco a momento y el sistema contraviento - disipador $(\alpha)$, y el sistema del disipador y contraviento $(\beta)$. Se aprovechan resultados de estudios previos (Nangullasmú 2011, Tena y Nangullasmú 2013, Tena-Colunga y Nangullasmú-Hernández 2015) y algunas consideraciones de la práctica profesional, para proponer diferentes parámetros geométricos estructurales, como alturas de los modelos y ángulos de inclinación para los contravientos $(\theta)$.

\section{DESCRIPCIÓN DE LOS MODELOS EVALUADOS}

Se analizaron marcos planos de concreto reforzado, con un sistema de contraventeo de acero de tipo chevrón que servirá de soporte a dispositivos disipadores de energía por histéresis del material. La configuración de los modelos en estudio corresponde a edificios regulares, formados por crujías de $8 \mathrm{~m}$ de claro, con alturas de entrepiso de $4 \mathrm{~m}$ y ángulo de inclinación de los contravientos con respecto al plano 
horizontal de $\theta=45^{\circ}$. Además, se variaron el número de niveles de los marcos en múltiplos de cinco, siendo el modelo más esbelto de 25 niveles. En la figura 1 con los colores se ilustra, de manera esquemática, los cambios de secciones transversales tipo conforme a la altura (usanza común en la práctica ingenieril mexicana). Se observa también que no coinciden cambios de secciones transversales del marco con los de contravientos en un mismo nivel para ninguno de los modelos analizados, con el objetivo de mitigar la posible formación de pisos débiles por cambios abruptos de rigidez que se pudieran presentar por dicho efecto.
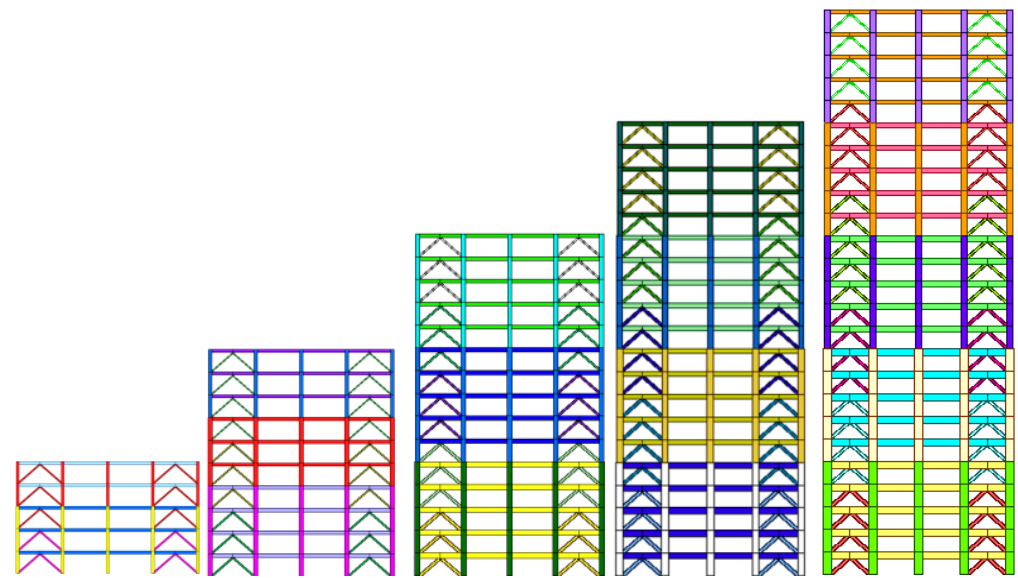

Figura 1. Representación de los cambios de secciones transversales para los modelos estudiados

El método evaluado en el presente trabajo toma balances de rigidez entre el sistema marco y contraviento-disipador $(\alpha)$ y balances de rigidez entre el contraviento y el disipador $(\beta)$, acorde a la metodología propuesta en trabajos previos (Nangullasmú 2011, Tena y Nangullasmú 2013, Tena-Colunga y Nangullasmú-Hernández 2015). Se evaluaron tres casos de balance de rigidez entre los sistemas estructurales marco y contraviento-disipador " $\alpha$ " $(\alpha=0.25$, el sistema del marco aporta menor rigidez lateral al sistema global; $\alpha=0.50$, el sistema del marco aporta la misma rigidez lateral al sistema global que el del sistema contraviento-disipador; y $\alpha=0.75$, el sistema del marco aporta mayor rigidez lateral al sistema global). Además, se proponen dos balances de rigidez elástica que deben tener los disipadores de energía con respecto a los contravientos de soporte, para que los disipadores alcancen su máxima capacidad de ductilidad $(\beta=1.0$, el contraviento es igual de rígido que el disipador y $\beta=0.50$, el disipador es la mitad de rígido que el contraviento).

Para todos los modelos evaluados, se definió un cortante sísmico de diseño común del $10 \%$ del peso total de la estructura $(\mathrm{V} / \mathrm{W}=0.10)$. La metodología de prediseño, basada en los balances de rigidez antes descritos, se expone con mayor detalle en Nangullasmú (2011) y Tena y Nangullasmú (2013). En la tabla 1 se presentan las rigideces laterales de cada uno de los sistemas involucrados para el modelo de 20 niveles. Los cambios de colores en las columnas distinguen las variaciones de las secciones transversales conforme a la altura del modelo estudiado. Se observa de esta tabla que el uso de secciones típicas en los elementos estructurales y la variación de las secciones transversales conforme la altura no permiten que el balance de rigidez se cumpla en todos los entrepisos con rigurosidad. Especialmente, se aprecia una mayor variación del parámetro " $\alpha$ " en los niveles donde existe el cambio de sección. Por cuestiones prácticas, se cuidó que este parámetro no tuviera una variación mayor al 10\%, y que se cumpliera en al menos el $75 \%$ de los entrepisos del marco, para tomar como válido el balance de rigidez entre el sistema del marco y el del contraviento-disipador $(\alpha)$. 
Además de vigilar que los balances de rigidez lateral entre los sistemas cumplieran con los parámetros propuestos inicialmente en la metodología de prediseño, se revisaron que los elementos estructurales cumplieran con los requisitos de resistencia que las Normas Técnicas Complementarias del Distrito Federal del 2004 exponen en cada uno de sus apartados para cada material involucrado (acero para contravientos y concreto para elementos del marco). Con base en esto, los contravientos se revisaron para que su relación de esbeltez no excediera el máximo permitido por la norma (NTCEM-04, Ap. 6.2.3.2), y que su resistencia de diseño estuviera dentro de los estados límites pertinentes (NTCEM-04, Ap. 3.2.2). Como la filosofía de diseño de este sistema estructural es que se desarrolle toda la no linealidad del sistema en los disipadores de energía, se prevé que los contravientos permanezcan elásticos durante todo el evento sísmico, con la finalidad de evitar que los contravientos se dañen y los disipadores se desprendan antes que alcancen su ductilidad objetivo. Para asegurar esta premisa, se utilizó un factor de seguridad de 1.5 en la revisión por resistencia para los contravientos, según recomendaciones del Ing. Enrique Martínez Romero (Martínez 1994).

Tabla 1. Rigideces laterales del modelo $20 \mathrm{~N}, \alpha=0.50, \beta=0.50$ (unidades: $\mathrm{t} / \mathrm{m}$ )

\begin{tabular}{|c|c|c|c|c|c|}
\hline Nivel & $K_{\text {marco }}$ & $\mathbf{K}_{\mathbf{c v}}$ & $\mathbf{K}_{\text {disipador }}$ & $\mathbf{K}_{\text {cv-disipador }}$ & $\alpha$ \\
\hline 1 & $127,513.44$ & $271,788.64$ & $135,894.32$ & $51,769.43$ & 0.71 \\
\hline 2 & $57,903.63$ & $271,788.64$ & $135,894.32$ & $51,769.43$ & 0.53 \\
\hline 3 & $57,903.63$ & $271,788.64$ & $135,894.32$ & $51,769.43$ & 0.53 \\
\hline 4 & $57,903.63$ & $271,788.64$ & $135,894.32$ & $51,769.43$ & 0.53 \\
\hline 5 & $57,903.63$ & $196,429.06$ & $98,214.53$ & $37,415.22$ & 0.61 \\
\hline 6 & $46,153.21$ & $196,429.06$ & $98,214.53$ & $37,415.22$ & 0.55 \\
\hline 7 & $39,144.57$ & $196,429.06$ & $98,214.53$ & $37,415.22$ & 0.51 \\
\hline 8 & $39,144.57$ & $196,429.06$ & $98,214.53$ & $37,415.22$ & 0.51 \\
\hline 9 & $39,144.57$ & $129,063.27$ & $64,531.63$ & $24,583.64$ & 0.61 \\
\hline 10 & $39,144.57$ & $129,063.27$ & $64,531.63$ & $24,583.64$ & 0.61 \\
\hline 11 & $29,915.39$ & $129,063.27$ & $64,531.63$ & $24,583.64$ & 0.55 \\
\hline 12 & $25,751.57$ & $129,063.27$ & $64,531.63$ & $24,583.64$ & 0.51 \\
\hline 13 & $25,751.57$ & $115,110.48$ & $57,555.24$ & $21,925.97$ & 0.54 \\
\hline 14 & $25,751.57$ & $115,110.48$ & $57,555.24$ & $21,925.97$ & 0.54 \\
\hline 15 & $25,751.57$ & $115,110.48$ & $57,555.24$ & $21,925.97$ & 0.54 \\
\hline 16 & $16,777.17$ & $115,110.48$ & $57,555.24$ & $21,925.97$ & 0.43 \\
\hline 17 & $12,928.90$ & $60,462.07$ & $30,231.04$ & $11,516.75$ & 0.53 \\
\hline 18 & $12,928.90$ & $60,462.07$ & $30,231.04$ & $11,516.75$ & 0.53 \\
\hline 19 & $12,928.90$ & $60,462.07$ & $30,231.04$ & $11,516.75$ & 0.53 \\
\hline 20 & $12,928.90$ & $60,462.07$ & $30,231.04$ & $11,516.75$ & 0.53 \\
\hline
\end{tabular}

Los elementos del marco se diseñaron conforme a lo establecido en las secciones 6.1 y 6.2 (NTCC04 2004), correspondientes al diseño de vigas y columnas de concreto reforzado no dúctiles, y se complementaron los requisitos antes mencionados con recomendaciones mínimas para detallado por confinamiento dúctil. En la sección 7.2.3, se especifica la separación mínima por confinamiento para elementos a flexión (vigas), mientras que en la sección 7.3.4 se establece la separación mínima de estribos por confinamiento de elementos sometidos a flexocompresión (columnas, figura 2), de las Normas Técnicas Complementarias para Diseño y Construcción de Estructuras de Concreto del 2004 (NTCC-04 
2004). El fundamento de adicionar estos requisitos mínimos para detallado por confinamiento dúctil a los elementos de concreto, diseñados como elementos no dúctiles, tiene su base en la dificultad que exponen los ingenieros estructurales en México para construir y diseñar marcos dúctiles siguiendo todas las pautas expuestas en las normas (Riobóo 1995, Tena-Colunga et al. 2008).

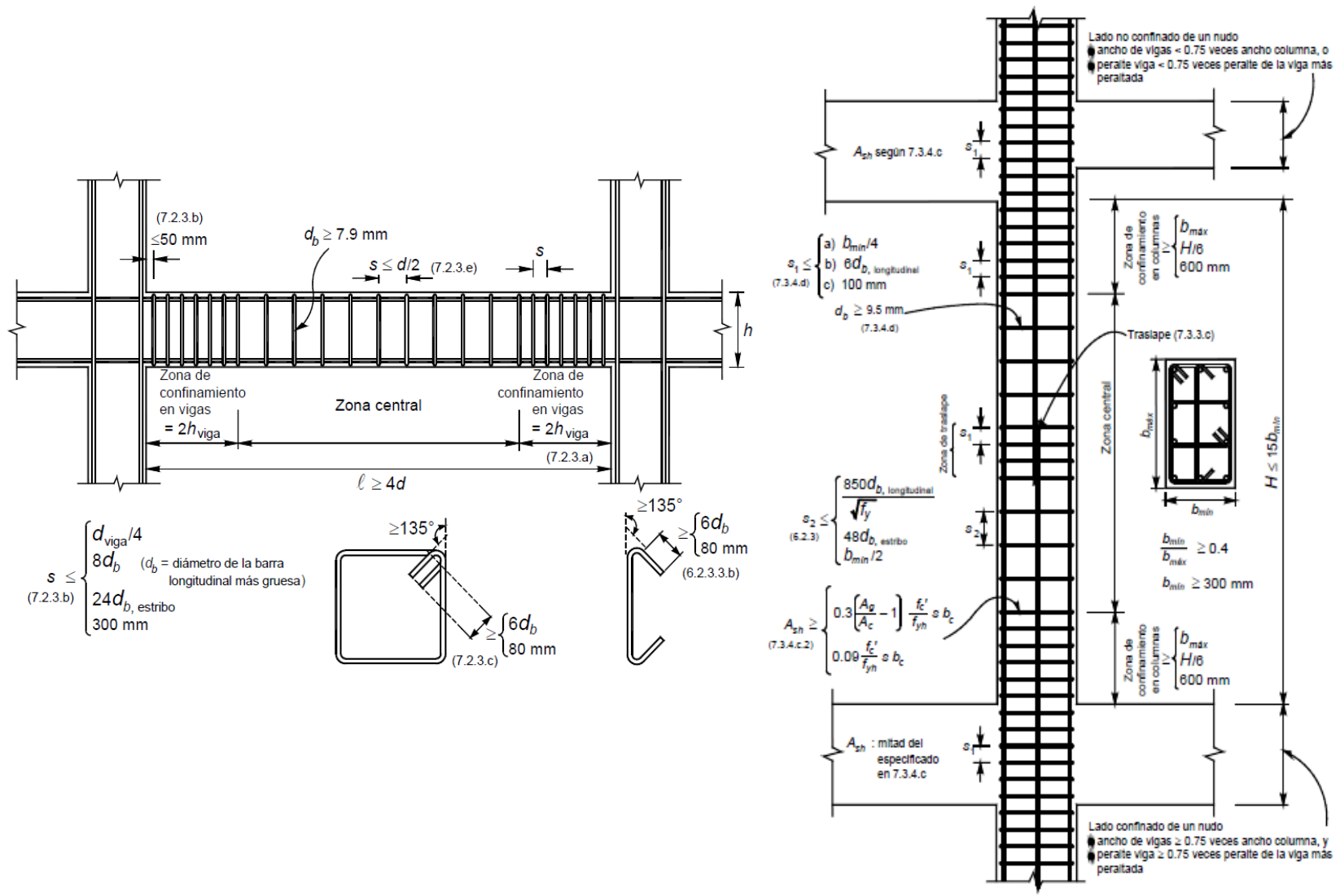

Figura 2. Detallado de elementos para marcos dúctiles (NTCC-04 2004)

\section{IMPACTO DEL CONFINAMIENTO EN ELEMENTOS DE CONCRETO REFORZADO}

Se empleó el programa BIAX (Wallace y Moehle 1989) para el modelado de los diagramas momento-curvatura y de interacción de los elementos de concreto reforzado. Este programa permite el modelado de la sección transversal por medio de un mallado, propuesto por el usuario, permitiendo la consideración de curvas esfuerzo-deformación para concreto confinado y sin confinar. El programa tiene la opción de utilizar, entre otros, los modelos constitutivos para el concreto descritos por Kent y Park (1986), además de permitir el modelado del acero de refuerzo como una curva bilineal con consideraciones de efectos de endurecimiento por deformación.

El presente trabajo utilizó la curva esfuerzo-deformación propuesta por Kent y Park, la cual se basa en evidencia experimental, y consta de una parte ascendente representada por una curva de segundo grado. El modelo supone que no se afecta a la deformación por esfuerzo máximo, pero si provoca un aumento en su resistencia (Park y Paulay 1986). El parámetro que define la rama descendente en línea recta está representado por la letra ' $Z$ ', y se especifica por la deformación presente cuando el esfuerzo ha caído hasta $0.5 \mathrm{f}$ ' . La ecuación que representa a esta variable está dada por: 
$Z=\frac{0.5}{\varepsilon_{50 u}+\varepsilon_{50 h}-0.003}$

La variable $\varepsilon_{50 \mathrm{~h}}$, proporciona la ductilidad adicional debida a los estribos, y relaciona el cociente de volúmenes entre el corazón de concreto confinado y el de los estribos utilizados.

$\varepsilon_{50 h}=\frac{3}{4} \rho_{s} \sqrt{\frac{b \prime \prime}{s_{h}}}$

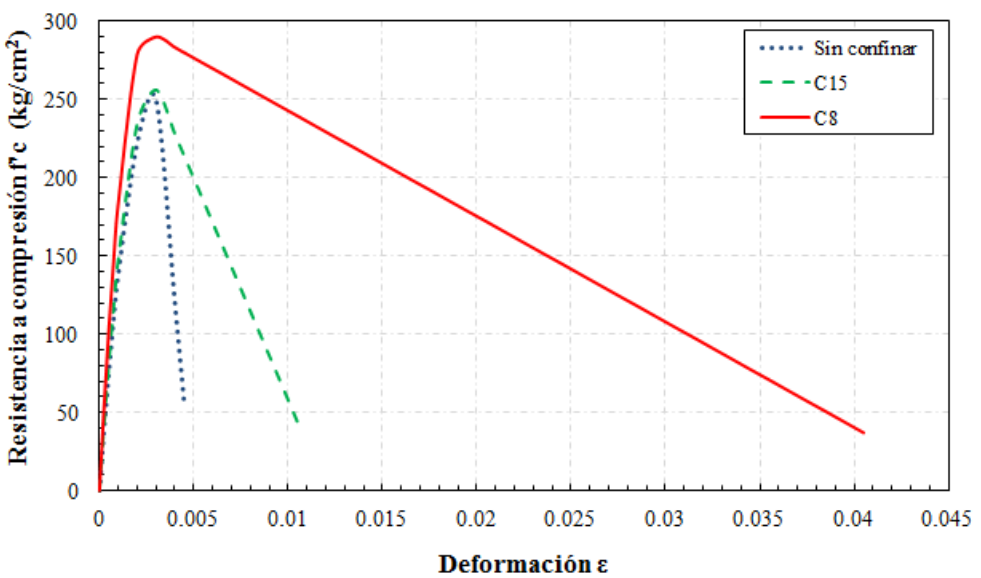

Figura 3. Curva esfuerzo-deformación para concreto confinado por estribos rectangulares

En la figura 3 están representadas tres curvas esfuerzo-deformación a distintos niveles de confinamiento. La curva con línea punteada representa una curva típica para un concreto sin confinar. La curva con línea discontinua representa a un elemento de concreto con separación de estribos a $15 \mathrm{cms}$. La curva con línea continua representa un elemento de concreto con separación máxima de estribos a $8 \mathrm{cms}$. Como se aprecia de esta figura, el considerar una separación de estribos más estrecha aumenta substancialmente la capacidad de deformación última y, en menor medida, su resistencia a compresión.

Con base en el soporte teórico que produce el efecto de confinamiento descrito con el modelo de Kent y Park, y modelando las secciones transversales con su respectivo refuerzo longitudinal y transversal, resultado del diseño descrito en la sección anterior, se comparan en la figura 4 los diagramas momento-curvatura para una columna de concreto con distintas separaciones de estribos. La línea discontinua representa un detallado no dúctil con una separación de estribos de $15 \mathrm{~cm}$ y la línea continua representa un detallado transversal dúctil según los requerimientos de las NTCC-04 (2004). De esta figura se observa que proveer a los elementos de concreto reforzado con las especificaciones mínimas de detallado dúctil, aumenta ligeramente su resistencia con respecto a la de un detallado no dúctil, pero incrementa notablemente su curvatura límite, siendo éste el parámetro más característico. En consecuencia, su capacidad de rotación incrementa de tres a cuatro veces con respecto a la de un detallado transversal no dúctil, y se verá reflejada con mayor detalle en la comparación de mapeos de fluencia que se exponen y discuten más adelante. 


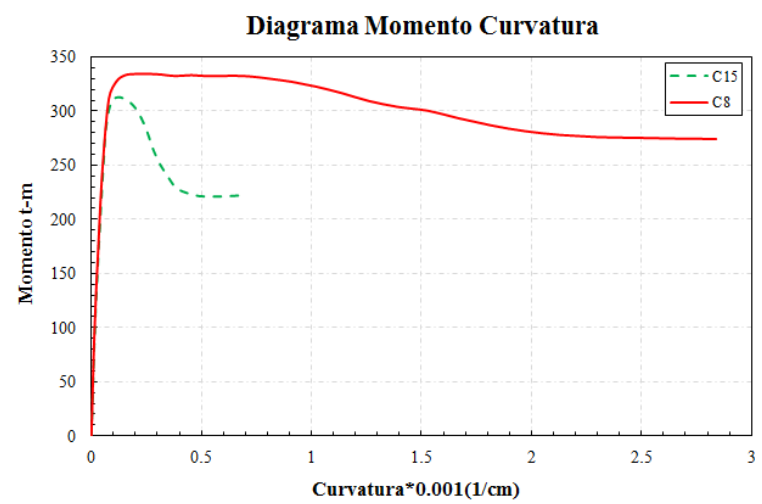

Figura 4. Diagrama momento-curvatura para distintas separación de estribos

Para las columnas, además del diagrama momento-curvatura, también se obtuvieron los diagramas de interacción momento - carga axial. En la figura 5 se presentan diagrama momento - carga axial para distintas separaciones de estribos, acorde al detallado para confinamiento dúctil (línea continua) y detallado no dúctil (línea discontinua). De ésta se observa que la capacidad de resistencia del elemento aumenta conforme se hace más estrecha la separación de los estribos.

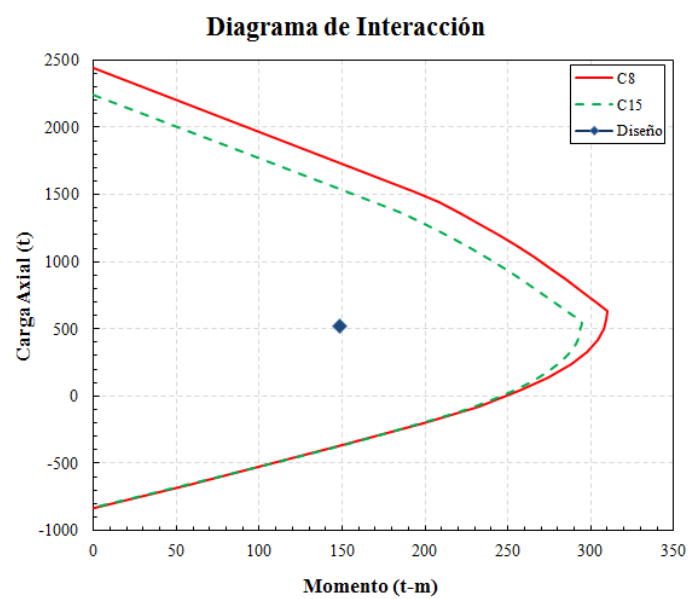

Figura 5. Diagrama momento- carga axial para distintas separaciones de estribos

El objetivo principal de adicionar requisitos mínimos de confinamiento dúctil a los marcos de concreto con disipadores de energía por histéresis del material, es analizar si ésto es suficiente para disminuir las magnitudes de rotación, representadas por una escala de colores (figura 6), que está asociado al daño moderado en vigas y columnas para marcos de concreto reforzado con detallado no dúctil y disipadores de energía (Nangullasmú 2011, Tena y Nangullasmú 2013, Tena-Colunga y NangullasmúHernández 2015). Usando los lineamientos de detallado no dúctil, se presentaban fluencias incipientes a moderadas en algunas columnas del perímetro de ciertos niveles, aún para los balances óptimos de rigidez entre los sistemas estructurales (figura 7). Es importante recalcar que para que este sistema estructural sea eficiente y aporte un beneficio adicional sobre un sistema estructural convencional, el daño se debe focalizar en los disipadores de energía, y que los elementos del marco deben permanecer prácticamente elásticos o con una no linealidad incipiente durante todo el evento sísmico. 


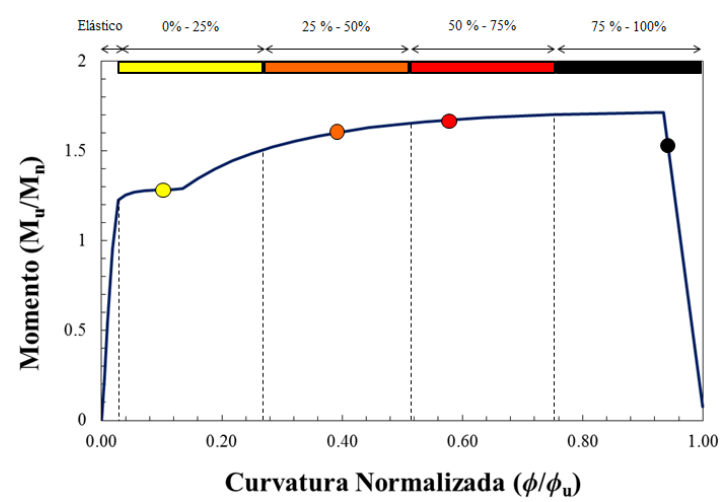

Figura 6. Escala de colores para el mapeo de fluencias inelásticas de vigas y columnas

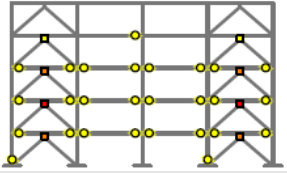

a) $\alpha=0.25, \beta=0.50$

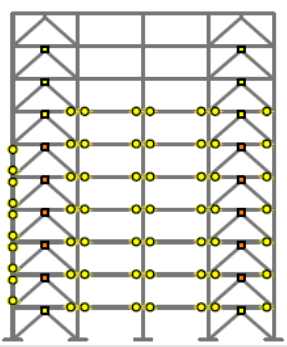

b) $\alpha=0.25, \beta=0.50$

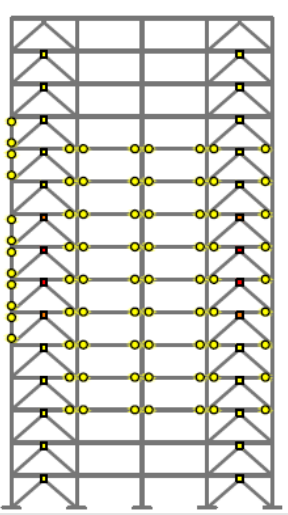

c) $\alpha=0.25, \beta=0.50$

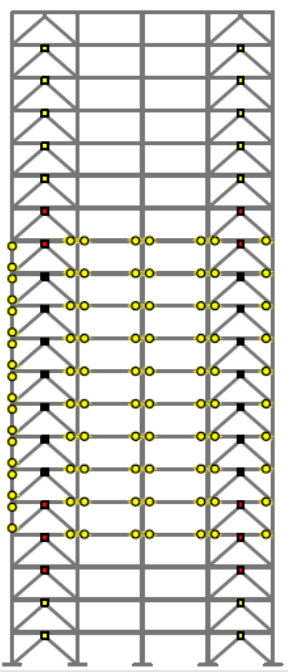

d) $\alpha=0.50, \beta=0.75$

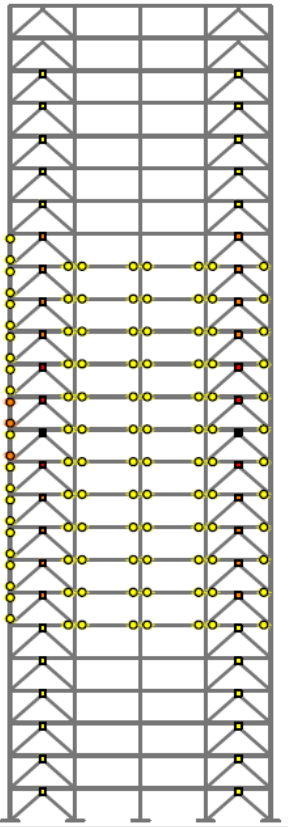

e) $\alpha=0.50, \beta=0.75$

Figura 7. Mapeo de rotaciones inelásticas para balances óptimos de rigidez cuando $\mathrm{Q} \leq 4$ (Tena-Colunga y Nangullasmú-Hernández 2015)

\section{DIFERENCIAS EN EL DISEÑO DEL REFUERZO POR CONFINAMIENTO}

En la presente sección se detallan las diferencias obtenidas del diseño de elementos de concreto reforzado usando los parámetros de marcos no dúctiles, comparado con el diseño adicionando los requisitos mínimos de confinamiento dúctil a los mismos elementos. Se aclara que para esta comparativa, el refuerzo longitudinal no presentó diferencia alguna, dado que los requisitos adicionales usados en dicho procedimiento se enfocan en el diámetro, número de ramas y separación de los estribos utilizados.

Los requisitos de refuerzo mínimo para marcos no dúctiles detallan que el diámetro a utilizarse en estribos no debe ser menor que el número 2.5 (Ap. 2.5.2.2 NTCC-04), y dado que las fuerzas cortantes para el diseño de los elementos del marco se reparten acorde al balance de rigidez a usar entre los 
diferentes sistemas, el usar estribos del número 2.5 (diámetro de $7.9 \mathrm{~mm}$ ), satisfizo para la mayoría de los casos las demandas de cortante (figuras 8,10 y 12), con excepción de las vigas para los modelos más esbeltos cuando el aporte de rigidez del marco era mayor $(\alpha=0.75)$, como se aprecia en la figura 14 .

En el detallado por confinamiento dúctil, existe una diferencia acerca del menor diámetro a utilizar en estribos para miembros a flexión y a flexocompresión. Para los elementos a flexión se especifica, al igual que para marcos no dúctiles, que el menor diámetro posible a utilizar en estribos es del No. 2.5 (Ap. 7.2.3 y 7.2.4.2 NTCC-04); mientras que para los elementos a flexocompresión, define que el refuerzo transversal debe estar formado por estribos cerrados con diámetro no menor al No. 3 o 9.5 mm (Ap. 7.3.4 NTCC-04). Estos requisitos hacen que las vigas no presenten diferencia en el diámetro utilizado por los estribos entre el diseño para marcos no dúctiles y la adición de los requisitos por confinamiento dúctil.

De manera esquemática, se aprecia que los estribos de las vigas, contenidas en las figuras 8 a 13, se encuentren representados de color rojo para los estribos No. 2.5 usado para dichos elementos. Para demandas grandes de cortante en los modelos esbeltos, el color de los estribos en vigas está representado en color azul (estribos No. 3), como se aprecia en las figuras 14 y 15. Además, con el procedimiento de diseño para marcos no dúctiles, en todas las columnas de todos los modelos se usaron estribos No. 2.5. La adición de los requisitos mínimos por confinamiento dúctil obligó a que la mayoría de las columnas utilizaran estribos del No. 3 (color azul), con diferencias en las columnas del último nivel de las figuras 9, 13 y 15, donde se necesitan de estribos del No. 4 (color verde), para satisfacer la condición de cuantía mínima de refuerzo transversal indicada en el Ap. 7.3.4c, cuando se usa la separación mínima y el número máximo posible de ramas entre estribos.

Tabla 2. Detallado de armado por confinamiento del modelo $15 \mathrm{~N}, \alpha=0.25, \beta=0.50$ (unidades: $\mathrm{cm}$ )

\begin{tabular}{|c|c|c|c|c|c|c|c|}
\hline & \multirow{2}{*}{ Entrepisos } & \multirow{2}{*}{ b } & \multirow{2}{*}{$\mathbf{h}$} & \multicolumn{2}{|c|}{ Confinamiento en los Extremos } & \multicolumn{2}{|c|}{ Confinamiento en el Centro } \\
\hline & & & & No Dúctil & Dúctil & No Dúctil & Dúctil \\
\hline \multirow{3}{*}{ Vigas } & V 1-5 & 60 & 90 & 4 No.2.5@30 & 6 No.2.5@15 & 4 No.2.5@35 & 6 No.2.5@20 \\
\hline & V 6-10 & 50 & 75 & 3 No.2.5 @ 25 & 6 No.2.5@15 & 3 No.2.5@30 & 6No.2.5@20 \\
\hline & V 11-15 & 40 & 65 & 3 No.2.5@30 & 4 No.2.5@15 & 3 No.2.5@35 & 4 No.2.5@20 \\
\hline \multirow{3}{*}{ Columnas } & C $1-5$ & 90 & 90 & 6 No.2.5@15 & 6 No.3@8 & 6 No.2.5@30 & 6 No.3@15 \\
\hline & C 6-10 & 80 & 80 & 6 No.2.5@15 & 6 No.3 @ 10 & 6 No.2.5@30 & 6 No.3 @ 15 \\
\hline & C $11-15$ & 70 & 70 & 4 No.2.5@12.5 & 4 No.4 @ 10 & 4 No.2.5@25 & 4 No. 4 @ 15 \\
\hline
\end{tabular}

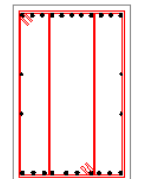

V $1-5$

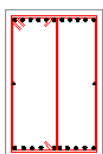

V $6-10$

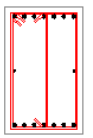

V $11-15$

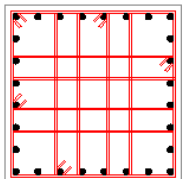

C $1-5$

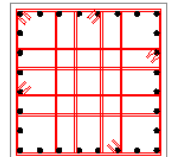

C $6-10$

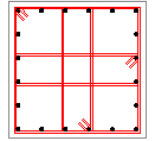

C $11-15$

Figura 8. Armado de elementos para modelo de 15 niveles, $\alpha=0.25, \beta=0.50$, confinamiento NO dúctil

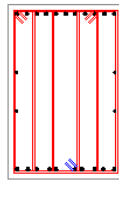

V $1-5$

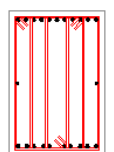

V $6-10$

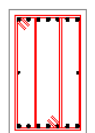

V $11-15$

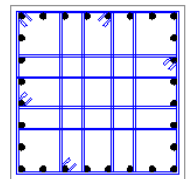

C $1-5$

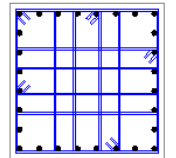

C $6-10$

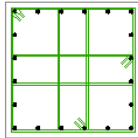

C $11-15$

Figura 9. Armado de elementos para modelo de 15 niveles, $\alpha=0.25, \beta=0.50$, confinamiento dúctil 
Tabla 3. Detallado de armado por confinamiento del modelo $15 \mathrm{~N}, \alpha=0.75, \beta=0.50$ (unidades: $\mathrm{cm}$ )

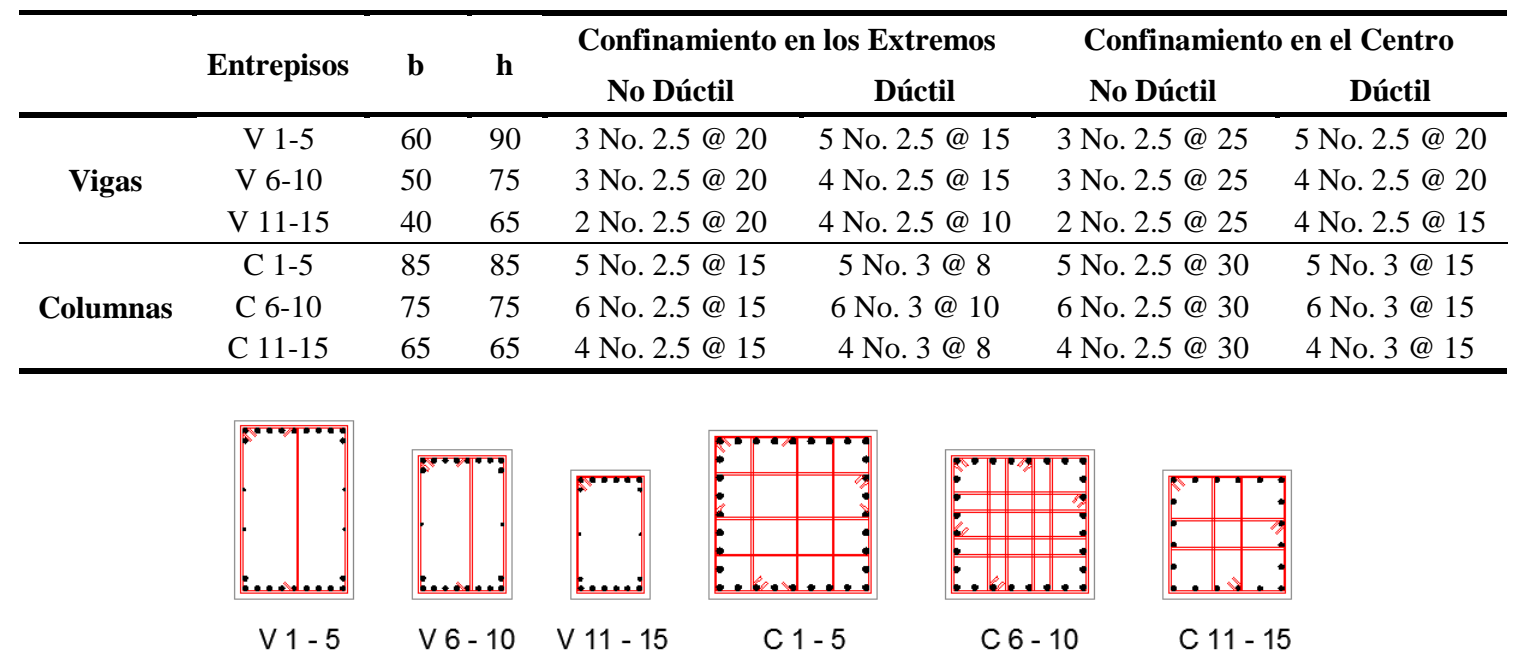

Figura 10. Armado de elementos para modelo de 15 niveles, $\alpha=0.75, \beta=0.50$, confinamiento NO dúctil
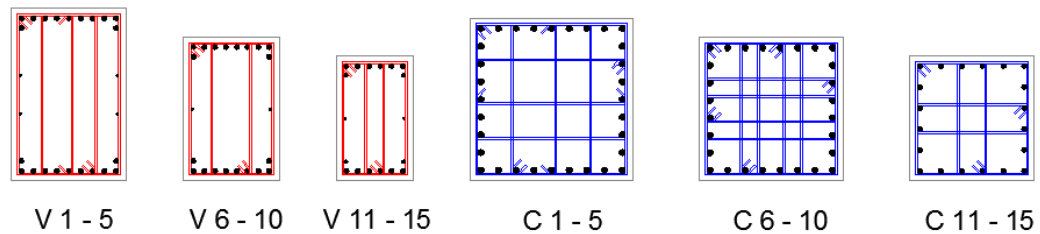

Figura 11. Armado de elementos para modelo de 15 niveles, $\alpha=0.75, \beta=0.50$, confinamiento dúctil

Tabla 4. Detallado de armado por confinamiento del modelo $25 \mathrm{~N}, \alpha=0.25, \beta=0.50$ (unidades: $\mathrm{cm}$ )

\begin{tabular}{|c|c|c|c|c|c|c|c|}
\hline & \multirow{2}{*}{ Entrepisos } & \multirow{2}{*}{ b } & \multirow{2}{*}{$\mathbf{h}$} & \multicolumn{2}{|c|}{ Confinamiento en los Extremos } & \multicolumn{2}{|c|}{ Confinamiento en el Centro } \\
\hline & & & & No Dúctil & Dúctil & No Dúctil & Dúctil \\
\hline \multirow{5}{*}{ Vigas } & V 1-5 & 75 & 110 & 3 No.3@25 & 6 No.3@15 & 3 No.3@30 & 6 No.3@20 \\
\hline & V 6-10 & 65 & 100 & 4 No.2.5@25 & 6 No.2.5@15 & 4 No.2.5@30 & 6 No.2.5@20 \\
\hline & V 11-15 & 55 & 85 & 3 No.2.5@25 & 5 No.2.5@15 & 3No.2.5@30 & 5 No.2.5@20 \\
\hline & V 16-20 & 45 & 70 & 3 No.2.5@25 & 4 No.2.5@15 & 2 No.2.5@30 & 4 No.2.5@20 \\
\hline & V 21-25 & 35 & 55 & 2 No.2.5@20 & 3No.2.5@10 & 2 No.2.5@25 & 3No.2.5@15 \\
\hline \multirow{5}{*}{ Columnas } & C $1-5$ & 150 & 150 & 13 No. $2.5 @ 15$ & 13 No. $3 @ 10$ & 13 No.2.5@35 & 13 No.3@15 \\
\hline & C 6-10 & 135 & 135 & 12 No. 2.5 @ 20 & 12 No. $3 @ 10$ & 12 No.2.5@35 & 12 No.3@15 \\
\hline & C $11-15$ & 120 & 120 & 10 No. 2.5 @ 20 & 10 No. 3 @ 10 & 10 No.2.5@35 & 10 No.3@15 \\
\hline & C $16-20$ & 105 & 105 & 8No.2.5 @15 & 8No.3@10 & 8 No.2.5@ 30 & 8 No.3@15 \\
\hline & C $21-25$ & 90 & 90 & 4 No.2.5@12.5 & 4 No.4@10 & 4 No.2.5@25 & 4 No.4@15 \\
\hline
\end{tabular}




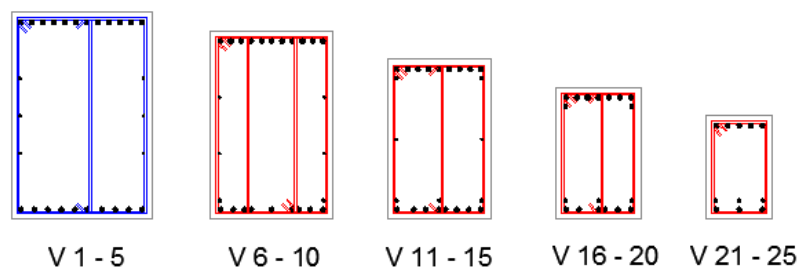

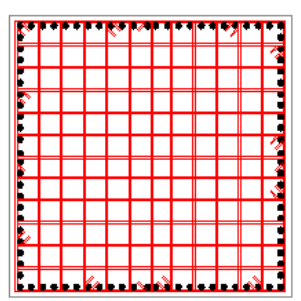

C 1 - 5

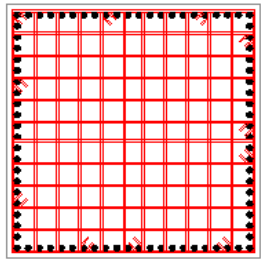

C $6-10$

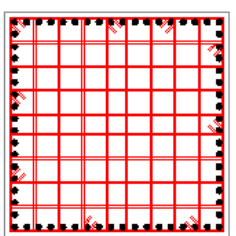

C $11-15$

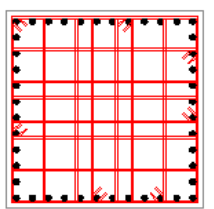

C 16 - 20

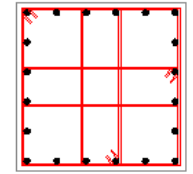

C 21 - 25

Figura 12. Armado de elementos para modelo de 25 niveles, $\alpha=0.25, \beta=0.50$, confinamiento NO dúctil
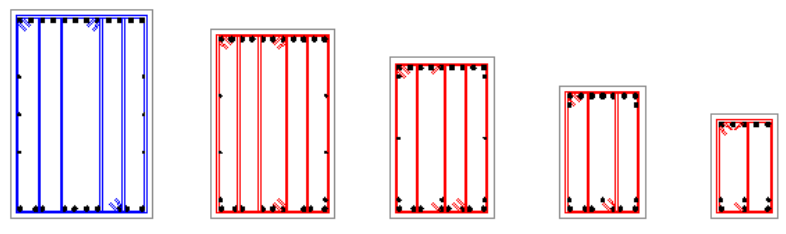

V 1 - 5

V 6 - 10

V $11-15$

V $16-20 \quad \vee 21-25$

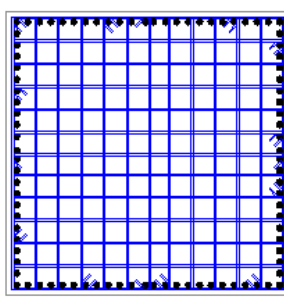

C 1 - 5

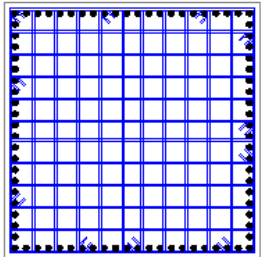

C $6-10$

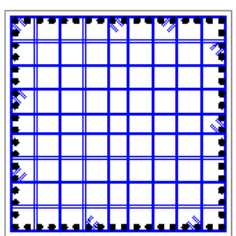

C $11-15$

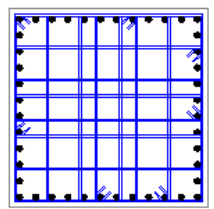

C $16-20$

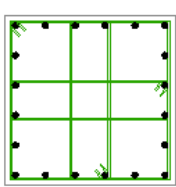

C $21-25$

Figura 13. Armado de elementos para modelo de 25 niveles, $\alpha=0.25, \beta=0.50$, confinamiento dúctil

Tabla 5. Detallado de armado por confinamiento del modelo $25 \mathrm{~N}, \alpha=0.75, \beta=0.50$ (unidades: $\mathrm{cm}$ )

\begin{tabular}{|c|c|c|c|c|c|c|c|}
\hline & \multirow{2}{*}{ Entrepisos } & \multirow{2}{*}{$\mathbf{b}$} & \multirow{2}{*}{$\mathbf{h}$} & \multicolumn{2}{|c|}{ Confinamiento en los Extremos } & \multicolumn{2}{|c|}{ Confinamiento en el Centro } \\
\hline & & & & No Dúctil & Dúctil & No Dúctil & Dúctil \\
\hline \multirow{5}{*}{ Vigas } & V 1-5 & 85 & 125 & 6 No.3@25 & 8 No.3@15 & 6 No.3@30 & 8 No.3@20 \\
\hline & V 6-10 & 75 & 115 & 6 No.3@25 & 7 No.3 @20 & 6 No.3@30 & 7 No.3@25 \\
\hline & V 11-15 & 70 & 105 & 4 No.3 @ 20 & 6 No.3@15 & 4 No.3 @ 25 & 6 No.3@20 \\
\hline & V 16-20 & 60 & 90 & 4 No.3 @ 25 & 6 No.3@15 & 4 No.3@30 & 6 No.3@20 \\
\hline & V 21-25 & 50 & 75 & 2 No.3@25 & 4 No.3@15 & 2 No.3@30 & 4 No.3@20 \\
\hline \multirow{5}{*}{ Columnas } & C $1-5$ & 145 & 145 & 13 No. $2.5 @ 15$ & 13 No. $3 @ 10$ & 13 No.2.5@35 & 13 No.3@15 \\
\hline & C 6-10 & 130 & 130 & 12 No. $2.5 @ 15$ & 12 No. $3 @ 10$ & 12 No.2.5@35 & 12 No.3@15 \\
\hline & C $11-15$ & 120 & 120 & 10 No.2.5@20 & 10 No. $3 @ 10$ & 10 No.2.5@35 & 10 No.3@15 \\
\hline & C $16-20$ & 105 & 105 & 8 No.2.5@20 & 8 No.3@10 & 8 No. 2.5 @ 35 & 8 No.3 @ 15 \\
\hline & C $21-25$ & 90 & 90 & 4 No. $2.5 @ 20$ & 4 No.4 @ 10 & 4 No. $2.5 @ 35$ & 4 No. $4 @ 15$ \\
\hline
\end{tabular}




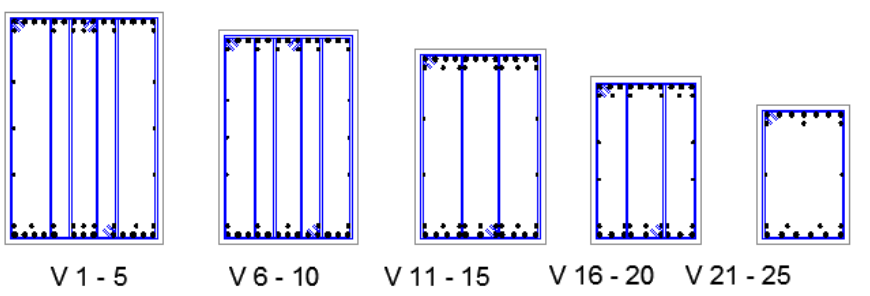

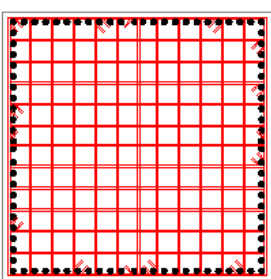

C 1 - 5

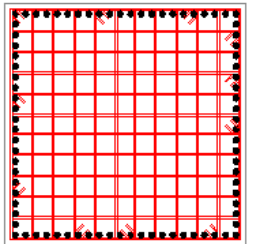

C $6-10$

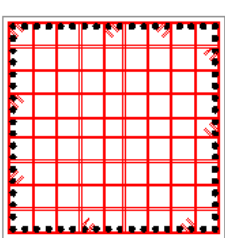

C $11-15$

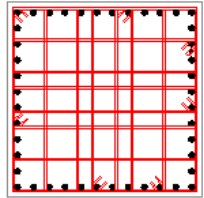

C $16-20$

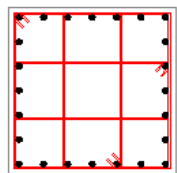

C $21-25$

Figura 14. Armado de elementos para modelo de 25 niveles, $\alpha=0.75, \beta=0.50$, confinamiento NO dúctil

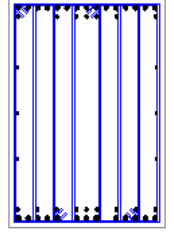

V 1 - 5

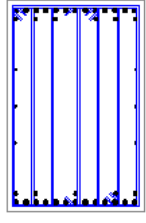

V 6 - 10
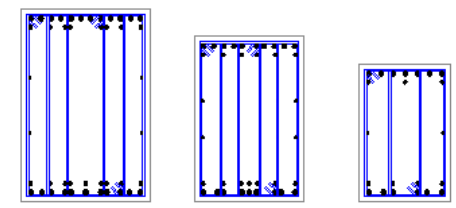

V $11-15 \quad \mathrm{~V} 16-20 \quad \mathrm{~V} 21-25$

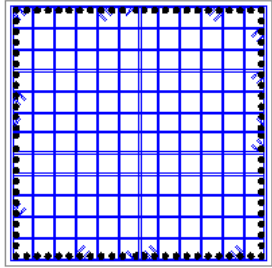

C $1-5$

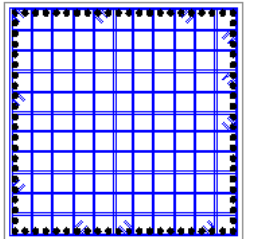

C $6-10$

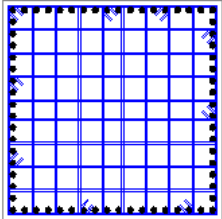

C $11-15$

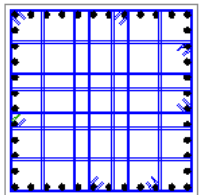

C $16-20$

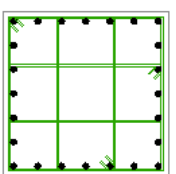

C $21-25$

Figura 15. Armado de elementos para modelo de 25 niveles, $\alpha=0.75, \beta=0.50$, confinamiento dúctil

El diámetro utilizado en los estribos es una diferencia importante en la adición de los requisitos de diseño por confinamiento dúctil, sobre todo en columnas. Sin embargo, si se observa el número de ramas de estribos utilizado en los armados por confinamiento no dúctil y dúctil en columnas, éstas son muy similares (tablas 2 a 5), y se debe a la condición de detallado del Ap. 6.2.3.3, que especifica que los estribos se dispondrán de manera que cada barra longitudinal de esquina y una de cada dos consecutivas de la periferia tenga un soporte lateral suministrado por el doblez de un estribo (NTCC-04 2004). Para el caso de las vigas, en los requisitos de detallado para marcos no dúctiles, no se especifica el uso del apartado anteriormente descrito. Debido a esto, las vigas con detallado no dúctil tienen menor número de ramas de estribos (figuras 8,10 y 12), que las vigas con detallado por confinamiento dúctil (figuras $9,11 \mathrm{y}$ 13), que en su Ap. 7.2.3d especifica el cumplimiento del Ap. 6.2.3.3, asociado al número de ramas de estribos a utilizar.

Como se aprecia en las tablas 2 a 5 , el parámetro crucial y que hace mayor diferencia entre el uso de confinamiento no dúctil y confinamiento dúctil es la separación de los estribos. Para la condición de confinamiento no dúctil en vigas, la separación de los estribos está condicionada a los Aps. 2.5.2.2 y 2.5.2.3 (NTCC-04 2004), y aunado al hecho de la distribución de la fuerza cortante de diseño entre el 
sistema del marco y del sistema contraviento-disipador, las separaciones entre los estribos son suficientemente holgadas para satisfacer la demanda de fuerza cortante (figura 16). Para el requisito de confinamiento dúctil en vigas, la separación máxima de los estribos en la zona de los extremos del miembro se encuentra delimitada por las condicionantes del Ap. 7.2.3b, del cual el que rige, en la mayoría de los casos, es el valor asociado a 24 veces el diámetro de la barra del estribo utilizada. Por lo tanto, para ciertos balances y condiciones de diseño, la diferencia en la separación de los estribos puede reducirse a la mitad, si se usan los requisitos de detallado por confinamiento dúctil, como se aprecia en la figura 17.

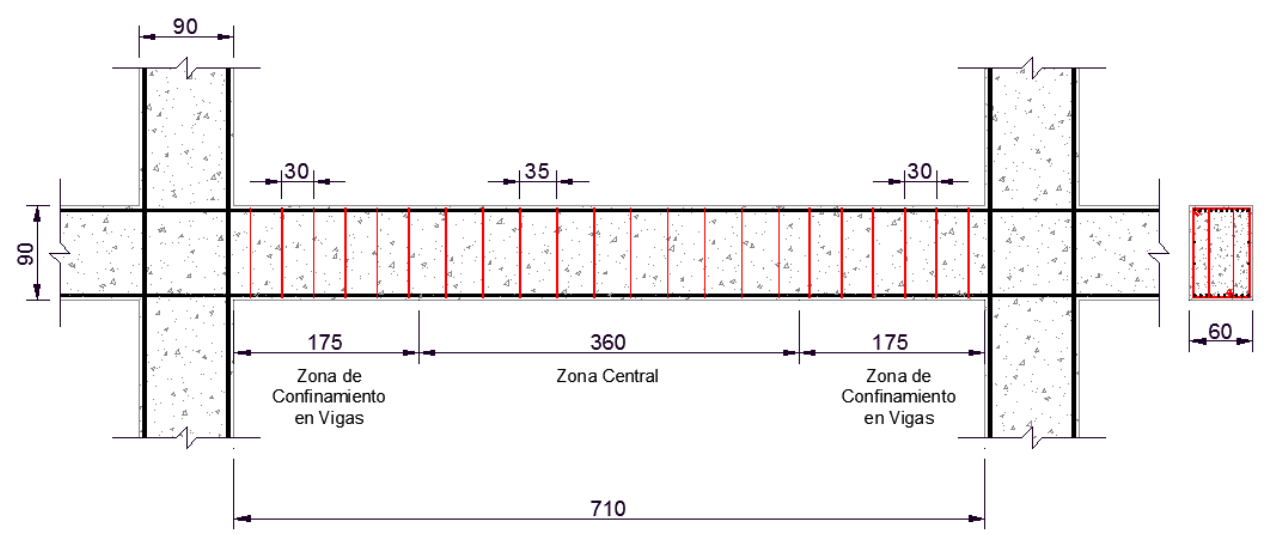

Figura 16. Detallado de elementos a flexión por confinamiento NO dúctil, modelo de 15 niveles con $\alpha=0.25, \beta=0.50$

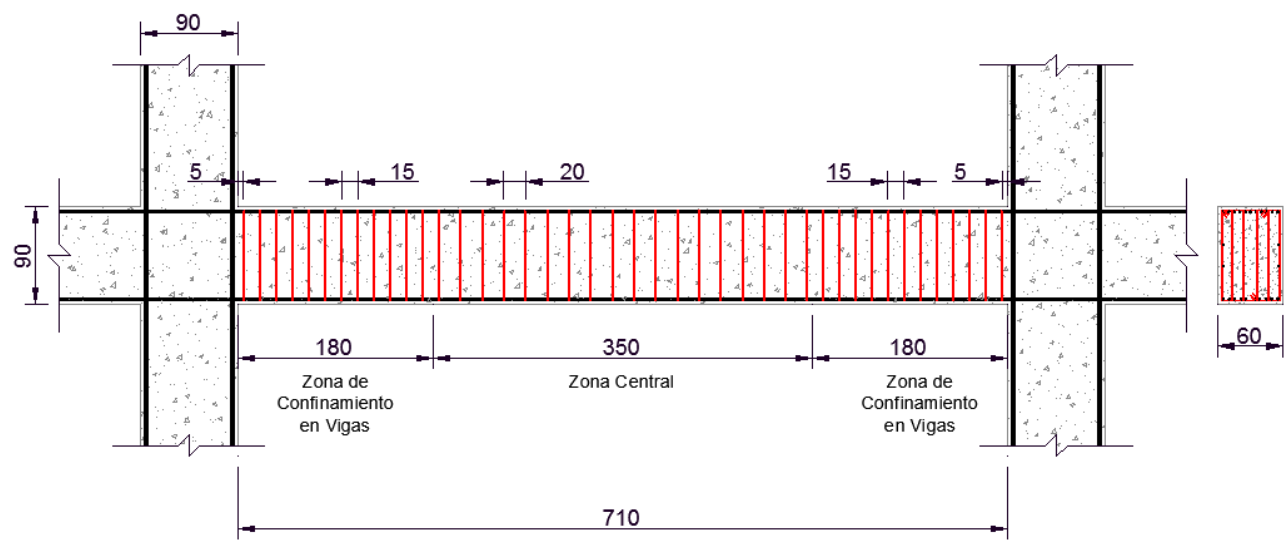

Figura 17. Detallado de elementos a flexión por confinamiento dúctil, modelo de 15 niveles con $\alpha=0.25$, $\beta=0.50$

En el caso de la separación de los estribos en columnas, para la condición de diseño por confinamiento no dúctil (figura 18), ésta estuvo determinada por el requisito de separación máxima permisible indicada en el Ap. 6.2.3.2 (NTCC-04). Lo anterior se debe, principalmente, a que las secciones transversales de las columnas son lo suficientemente robustas y, asociado a su armado longitudinal, cubren la demanda de cortante para elementos a flexocompresión que se especifica en el Ap. 2.5.1.3 (NTCC-04).

Para los requisitos por confinamiento dúctil de elementos a flexocompresión (figura 19), la separación del refuerzo transversal se definió acorde al Ap. 7.3.4 (NTCC-04), dónde se establece que no se debe exceder de la cuarta parte de la menor dimensión transversal del elemento, seis veces el diámetro 
de la barra longitudinal más gruesa o de $10 \mathrm{~cm}$. Para este caso de estudio, dicha separación no excedió de $10 \mathrm{~cm}$ para la condición de confinamiento dúctil, dado que para obtener una separación menor, se debería utilizar una sección transversal menor de $40 \mathrm{~cm}$ o armados longitudinales con varillas de diámetro de 5/8" o menores, que no se utilizaron para ningún balance de rigidez evaluado. Además, fueron pocos los casos donde la separación usada fue menor de $10 \mathrm{~cm}$ (Tabla 3), sobre todo donde disminuir la separación entre los estribos era más eficiente que aumentar el diámetro, o colocar una rama extra de estribo (figura 11).

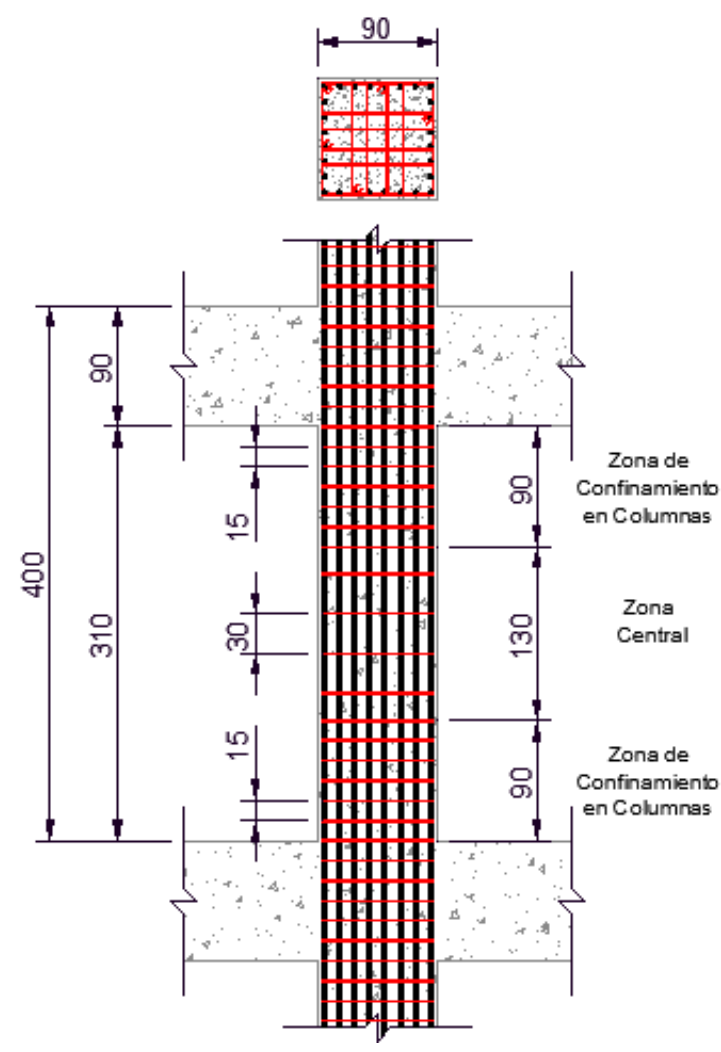

Figura 18. Detallado de elementos a flexocompresión por confinamiento NO dúctil, modelo de 15 niveles con $\alpha=0.25, \beta=0.50$

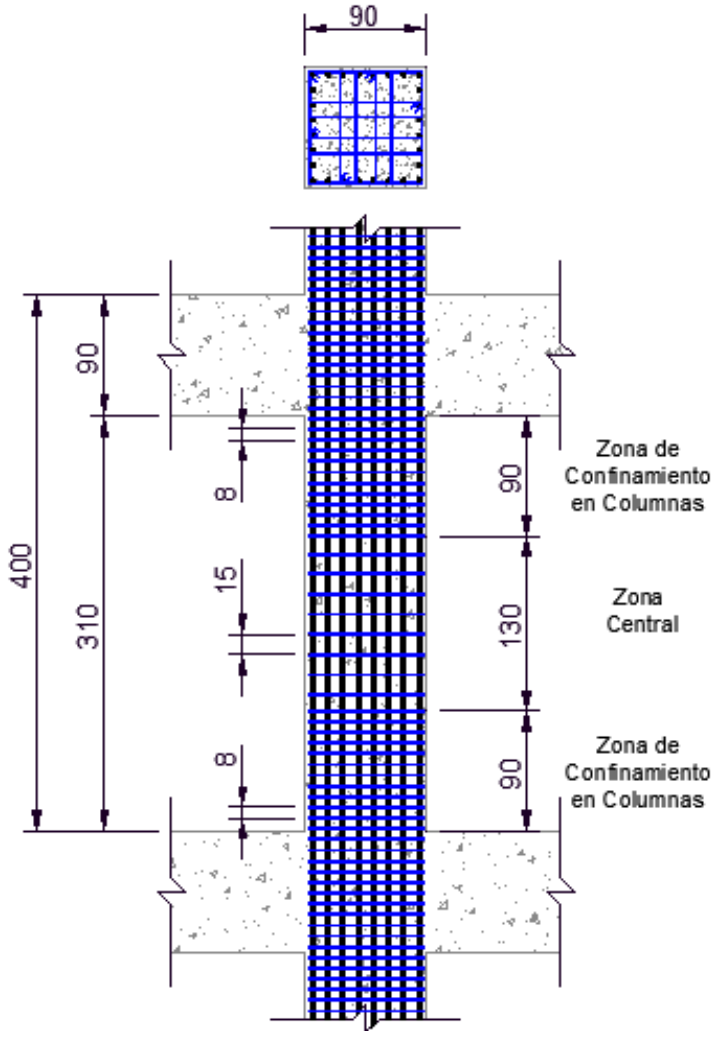

Figura 19. Detallado de elementos a flexocompresión por confinamiento dúctil, modelo de 15 niveles con $\alpha=0.25, \beta=0.50$

El uso de los requisitos mínimos para el confinamiento dúctil podría suponer un gran aumento en el presupuesto directo por insumos que representa la disminución en la separación entre los estribos, el aumento del diámetro mínimo para utilizar en columnas, o por el uso de las ramas necesarias para cumplir con las especificaciones solicitadas por la reglamentación mexicana en dicha cuestión. Por esta razón, se realizó una cuantificación de la cantidad de insumos necesarios para la construcción de los elementos que conforman el sistema del marco de los modelos evaluados. En primera instancia, se compara la diferencia en pesos del uso de detallado transversal con las especificaciones de marcos no dúctiles contra el detallado transversal dúctil. Como comparativa final, se utiliza el costo directo de la construcción de todos los elementos del marco.

En la tabla 6 se resumen los pesos cuantificados en toneladas del acero transversal y longitudinal, utilizados en los diferentes balances de rigidez. Se aprecia que el uso de detallado transversal para confinamiento dúctil implica un aumento de más de 2.5 veces en peso, del refuerzo transversal exclusivamente, para la mayoría de los modelos evaluados, comparado contra el no usar dicha adición en el procedimiento de diseño convencional para marcos no dúctiles. La mayor diferencia se presenta para el 
modelo de 15 niveles con balance de rigidez $\alpha=0.50$, donde el diámetro del estribo utilizado en columnas paso del número 2.5 al número 4 para todas las columnas de dicho modelo.

Tabla 6. Cuantificación de acero de refuerzo transversal y longitudinal de elementos de concreto

\begin{tabular}{|c|c|c|c|c|c|c|c|c|}
\hline \multirow{2}{*}{ Niveles } & \multirow{2}{*}{$\begin{array}{c}\text { Balances de } \\
\text { Rigidez }\end{array}$} & \multicolumn{2}{|c|}{$\begin{array}{l}\text { Rfzo. Transversal } \\
\text { (tonelada) }\end{array}$} & \multirow{2}{*}{ (\%) Peso } & \multirow{2}{*}{$\begin{array}{c}\text { Rfzo. } \\
\text { Longitudinal } \\
\text { (tonelada) }\end{array}$} & \multicolumn{2}{|c|}{$\begin{array}{c}\text { Rfzo. Transversal + } \\
\text { Longitudinal }(t)\end{array}$} & \multirow{2}{*}{ (\%) Peso } \\
\hline & & No Dúctil & Dúctil & & & No Dúctil & Dúctil & \\
\hline \multirow{3}{*}{5} & $\alpha=0.25$ & 7.2 & 21.4 & $295.6 \%$ & 63.1 & 70.4 & 84.5 & $120.1 \%$ \\
\hline & $\alpha=0.50$ & 9.2 & 27.1 & $293.1 \%$ & 69.8 & 79.0 & 96.9 & $122.6 \%$ \\
\hline & $\alpha=0.75$ & 12.2 & 35.7 & $293.4 \%$ & 80.6 & 92.8 & 116.3 & $125.3 \%$ \\
\hline \multirow{3}{*}{10} & $\alpha=0.25$ & 26.4 & 88.6 & $335.3 \%$ & 163.2 & 189.6 & 251.8 & $132.8 \%$ \\
\hline & $\alpha=0.50$ & 24.7 & 77.6 & $314.3 \%$ & 176.8 & 201.5 & 254.4 & $126.3 \%$ \\
\hline & $\alpha=0.75$ & 24.5 & 58.1 & $237.6 \%$ & 166.1 & 190.6 & 224.2 & $117.7 \%$ \\
\hline \multirow{3}{*}{15} & $\alpha=0.25$ & 41.1 & 125.4 & $305.4 \%$ & 288.7 & 329.7 & 414.1 & $125.6 \%$ \\
\hline & $\alpha=0.50$ & 35.9 & 134.9 & $375.8 \%$ & 341.3 & 377.2 & 476.2 & $126.3 \%$ \\
\hline & $\alpha=0.75$ & 38.9 & 102.7 & $264.2 \%$ & 389.3 & 428.2 & 492.1 & $114.9 \%$ \\
\hline \multirow{3}{*}{20} & $\alpha=0.25$ & 81.5 & 235.6 & $289.3 \%$ & 712.5 & 793.9 & 948.1 & $119.4 \%$ \\
\hline & $\alpha=0.50$ & 108.2 & 285.1 & $263.5 \%$ & 751.7 & 859.9 & 1036.8 & $120.6 \%$ \\
\hline & $\alpha=0.75$ & 117.8 & 329.0 & $279.4 \%$ & 781.1 & 898.8 & 1110.1 & $123.5 \%$ \\
\hline \multirow{3}{*}{25} & $\alpha=0.25$ & 167.3 & 479.2 & $286.5 \%$ & 1140.4 & 1307.7 & 1619.6 & $123.9 \%$ \\
\hline & $\alpha=0.50$ & 183.6 & 483.8 & $263.5 \%$ & 1163.6 & 1347.2 & 1647.4 & $122.3 \%$ \\
\hline & $\alpha=0.75$ & 230.3 & 577.9 & $251.0 \%$ & 1266.1 & 1496.3 & 1844.0 & $123.2 \%$ \\
\hline
\end{tabular}

Si se compara el peso total del acero de refuerzo utilizado (refuerzo transversal y refuerzo longitudinal), para columnas, vigas principales, vigas secundarias y losas de concreto, la diferencia en peso disminuye considerablemente. En promedio, el uso del detallado por confinamiento dúctil implica un aumento de apenas el $25 \%$ del peso total de acero de refuerzo cuantificado para los diversos modelos evaluados, aunque la variación en peso oscila en un intervalo entre el $15 \%$ y el $30 \%$, dependiendo del balance de rigidez usado. La menor diferencia en peso del acero de refuerzo se presentó para el modelo de 15 niveles con balance $\alpha=0.75$ (Tabla 3), a pesar que se aumentó el número de ramas de estribos usadas en vigas, además del aumento del diámetro utilizado para los estribos de las columnas (figura 10 y 11).

En la tabla 7 se presenta una comparativa de costos en insumos, y la variación del usar o no usar los requisitos mínimos de confinamiento dúctil para los elementos involucrados en la construcción del sistema del marco de las estructuras con disipadores de energía por histéresis del material. Los precios en esta tabla son precios comerciales durante el segundo semestre del año 2015 en la zona metropolitana de la Ciudad de México, con un costo de $\$ 1,867.60 / \mathrm{m}^{3}$ de concreto premezclado con resistencia a compresión de $\mathrm{f}^{\prime}{ }^{\prime}=250 \mathrm{~kg} / \mathrm{cm}^{2}$ y de $\$ 12,180.00 /$ ton para el acero de refuerzo con diámetros de No. 2.5 al No. 12. Dentro de la cuantificación se tomaron en cuenta, además de los elementos primarios del marco (vigas y columnas), la volumetría del sistema de piso planificado con losa maciza de concreto reforzado de $12 \mathrm{~cm}$ de espesor y de vigas secundarias para control de deflexiones. 
Tabla 7. Comparativa de precios entre el uso de detallado dúctil por confinamiento y no usarlo

\begin{tabular}{|c|c|c|c|c|c|c|c|}
\hline \multirow{2}{*}{ Niveles } & \multirow{2}{*}{$\begin{array}{l}\text { Balances de } \\
\text { Rigidez }\end{array}$} & \multirow{2}{*}{$\begin{array}{c}\text { Concreto } \\
\left(\mathbf{m}^{3}\right)\end{array}$} & \multicolumn{2}{|c|}{$\begin{array}{c}\text { Rfzo. Transversal + } \\
\text { Longitudinal }(\mathbf{t})\end{array}$} & \multicolumn{2}{|c|}{$\begin{array}{c}\text { Concreto + Acero de refuerzo } \\
(\$ \text { MXN) }\end{array}$} & \multirow{2}{*}{ (\%) Precio } \\
\hline & & & No Dúctil & Dúctil & No Dúctil & Dúctil & \\
\hline \multirow{3}{*}{5} & $\alpha=0.25$ & 809.4 & 70.4 & 84.5 & $\$ 2,368,597.09$ & $\$ 2,540,846.43$ & $107.27 \%$ \\
\hline & $\alpha=0.50$ & 858.8 & 79.0 & 96.9 & $\$ 2,566,498.38$ & $\$ 2,783,728.36$ & $108.46 \%$ \\
\hline & $\alpha=0.75$ & 930.4 & 92.8 & 116.3 & $\$ 2,867,718.34$ & $\$ 3,154,011.88$ & $109.98 \%$ \\
\hline \multirow{3}{*}{10} & $\alpha=0.25$ & 2275.5 & 189.6 & 251.8 & $\$ 6,559,361.89$ & $\$ 7,316,482.36$ & $111.54 \%$ \\
\hline & $\alpha=0.50$ & 2180.8 & 201.5 & 254.4 & $\$ 6,526,701.97$ & $\$ 7,170,907.27$ & $109.87 \%$ \\
\hline & $\alpha=0.75$ & 2275.5 & 190.6 & 224.2 & $\$ 6,570,612.83$ & $\$ 6,980,344.50$ & $106.24 \%$ \\
\hline \multirow{3}{*}{15} & $\alpha=0.25$ & 3535.7 & 329.7 & 414.1 & $\$ 10,619,379.24$ & $\$ 11,646,687.85$ & $109.67 \%$ \\
\hline & $\alpha=0.50$ & 3450.7 & 377.2 & 476.2 & $\$ 11,038,508.37$ & $\$ 12,244,833.44$ & $110.93 \%$ \\
\hline & $\alpha=0.75$ & 3450.7 & 428.2 & 492.1 & $\$ 11,660,131.80$ & $\$ 12,437,892.62$ & $106.67 \%$ \\
\hline \multirow{3}{*}{20} & $\alpha=0.25$ & 5552.7 & 793.9 & 948.1 & $\$ 20,040,068.00$ & $\$ 21,917,860.90$ & $109.37 \%$ \\
\hline & $\alpha=0.50$ & 6286.4 & 859.9 & 1036.8 & $\$ 22,214,207.19$ & $\$ 24,368,701.31$ & $109.70 \%$ \\
\hline & $\alpha=0.75$ & 6286.4 & 898.8 & 1110.1 & $\$ 22,688,307.79$ & $\$ 25,261,314.84$ & $111.34 \%$ \\
\hline \multirow{3}{*}{25} & $\alpha=0.25$ & 7931.9 & 1307.7 & 1619.6 & $\$ 30,741,331.19$ & $\$ 34,540,881.61$ & $112.36 \%$ \\
\hline & $\alpha=0.50$ & 8049.4 & 1347.2 & 1647.4 & $\$ 31,441,492.31$ & $\$ 35,098,154.99$ & $111.63 \%$ \\
\hline & $\alpha=0.75$ & 8960.5 & 1496.3 & 1844.0 & $\$ 34,959,803.00$ & $\$ 39,194,633.53$ & $112.11 \%$ \\
\hline
\end{tabular}

De manera general, se aprecia de la tabla 7 que los costos en insumos son mayores conforme el balance " $\alpha$ " va en aumento. Comparando los mismos requisitos de detallado para los diferentes balances de rigidez, se observa que para los modelos de 10 niveles la variación en costos es apenas perceptible. Además, comparando todos los modelos con sus distintos balances de rigidez, se distingue que el usar o no usar un detallado por confinamiento dúctil tiene una variación promedio en costos de insumo del $10 \%$, que oscila en un intervalo entre el $6 \%$ y el $12 \%$, siendo los modelos más esbeltos estudiados donde se aprecia la mayor diferencia en costos entre el tipo de detallado transversal utilizado.

\section{ANÁLISIS ESTÁTICOS NO LINEALES}

Se efectuaron análisis estáticos no lineales bajo cargas laterales monótonas crecientes con el programa DRAIN-2DX (Prakash et al. 1992). Se presentan resultados característicos para los diversos modelos evaluados con comparativas entre diagramas tomando en cuenta ductilidades locales de los disipadores $\left(\mu_{d}\right)$, curvas de cortante basal normalizado - distorsión global de azotea, y mapeos de fluencias para los distintos balances de rigidez evaluados. El objetivo principal es analizar si los requisitos de detallado por confinamiento dúctil, explicados en párrafos anteriores, son suficientes para hacer que el mecanismo estructural de estos sistemas sea más eficiente para los balances de rigidez evaluados en estos casos de estudio.

En la figura 20 se presentan los diagramas de deformación inelástica de los disipadores de energía para marcos con detallado por confinamiento dúctil. En el eje horizontal se define la ductilidad local desarrollada por cada uno de los disipadores utilizados para los entrepisos asociados en el eje vertical. Las líneas punteadas representan los límites entre los cuales los disipadores presentan un desempeño eficiente $\left(8 \leq \mu_{d} \leq 12\right)$. En esta figura se hace la comparación entre los diferentes aportes de rigidez del marco y del sistema contraviento-disipador $(\alpha)$, y de manera separada, la comparativa entre la variación de rigidez del 
disipador con respecto al contraviento de soporte $(\beta)$. De esta figura se aprecia que el balance en el cuál los disipadores desarrollan mayores ductilidades es cuando el marco aporta menor rigidez al sistema completo $(\alpha=0.25)$, dando oportunidad que más de la mitad de los disipadores de la estructura alcancen una ductilidad adecuada $\left(\mu_{d}=6.0\right)$. Además, se aprecia que conforme la rigidez del disipador es menor que la del contraviento $(\beta=0.50)$, las magnitudes de las ductilidades desarrolladas por los disipadores son mayores, especialmente cuando los elementos del marco aportan mayor rigidez al sistema global $(\alpha=0.75)$.
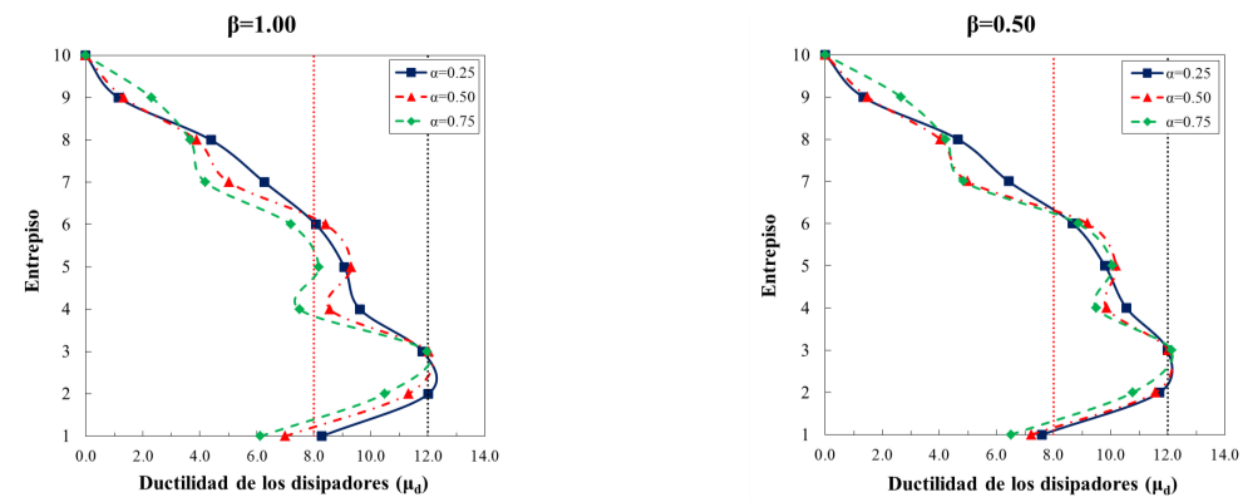

Figura 20. Comparativa de ductilidades de los disipadores $\mu_{\mathrm{d}}$ en modelos de 10 niveles con detallado por confinamiento dúctil
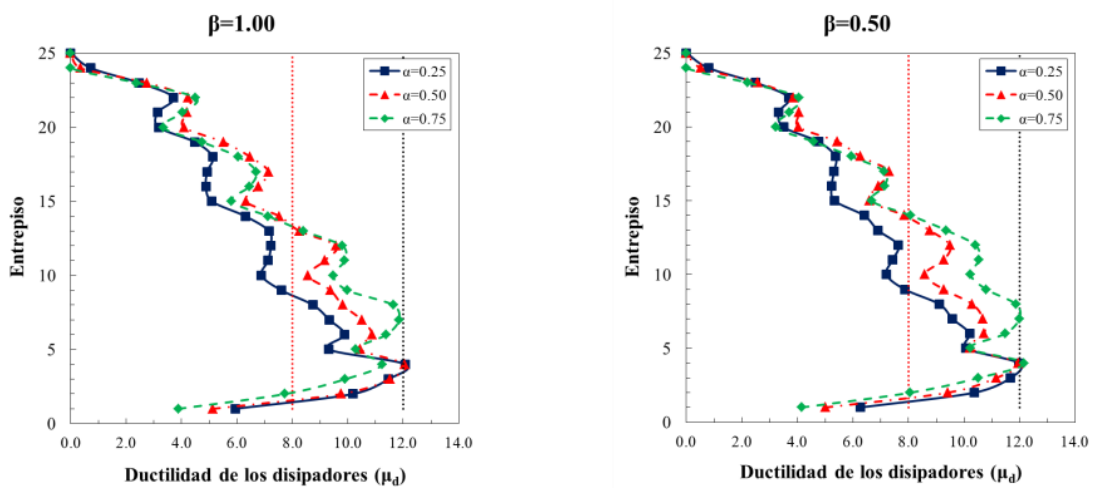

Figura 21. Comparativa de ductilidades de los disipadores $\mu_{\mathrm{d}}$ en modelos de 25 niveles con detallado por confinamiento dúctil

Conforme los modelos evaluados se van haciendo más esbeltos, el balance de rigidez " $\alpha$ ", donde más disipadores alcanzan una ductilidad adecuada, cambia a cuando el marco aporta mayor rigidez al sistema $(\alpha=0.50$ y $\alpha=0.75)$. En la figura 21 se observa que menos de la mitad de los disipadores utilizados alcanzan su ductilidad objetivo para el balance de " $\alpha=0.25$ ", sin importar el balance de rigidez " $\beta$ " utilizado. Se infiere a partir de estos diagramas que, para el balance antes mencionado, el sistema contraviento-disipador es demasiado rígido para que los disipadores desarrollen ductilidades " $\mu_{\mathrm{d}} \geq 8.0$ ", y aunado a mapeos de fluencias inelásticas, curvas de cortante basal normalizado y curvas de distorsión global de azotea, que se expondrán a detalle más adelante, se podrá apreciar el efecto que tiene la variación del parámetro “ $\alpha$ ” en las estructuras esbeltas.

En las figuras 22 a 24 se grafican las curvas de cortante basal normalizado-distorsión global de azotea para los modelos evaluados con detallado por confinamiento dúctil. En el eje de las ordenadas está representada la sobrerresistencia, definida como un cociente entre el cortante global desarrollado y su 
cortante de diseño. En el eje de las abscisas se presenta la distorsión global alcanzada por el sistema en términos de porcentaje.
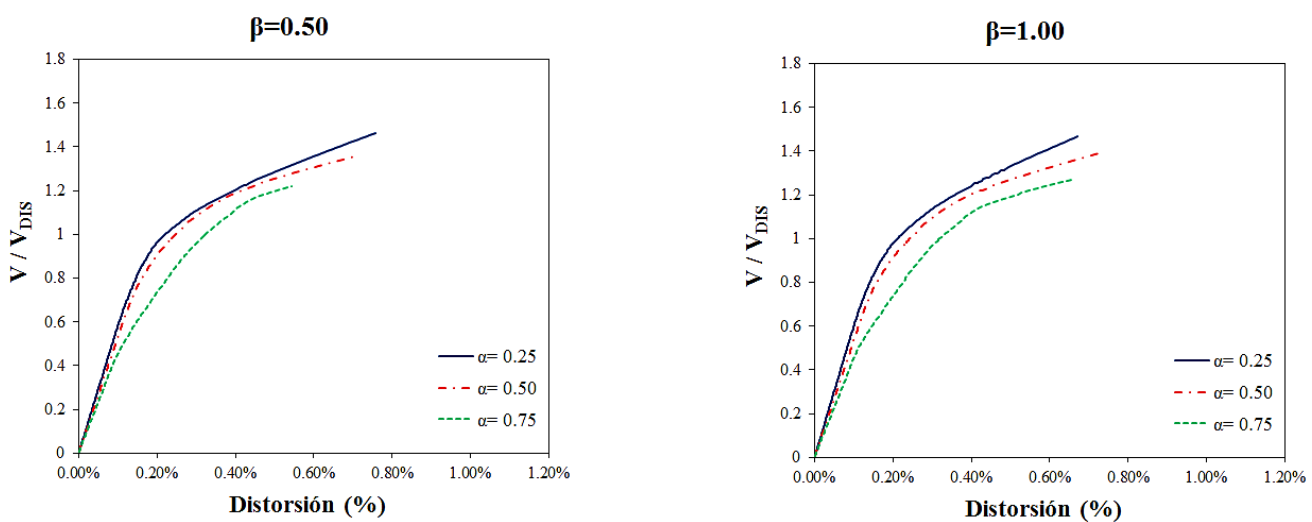

Figura 22. Curvas de cortante basal normalizado-distorsión global de azotea, modelos de 15 niveles con detallado por confinamiento dúctil
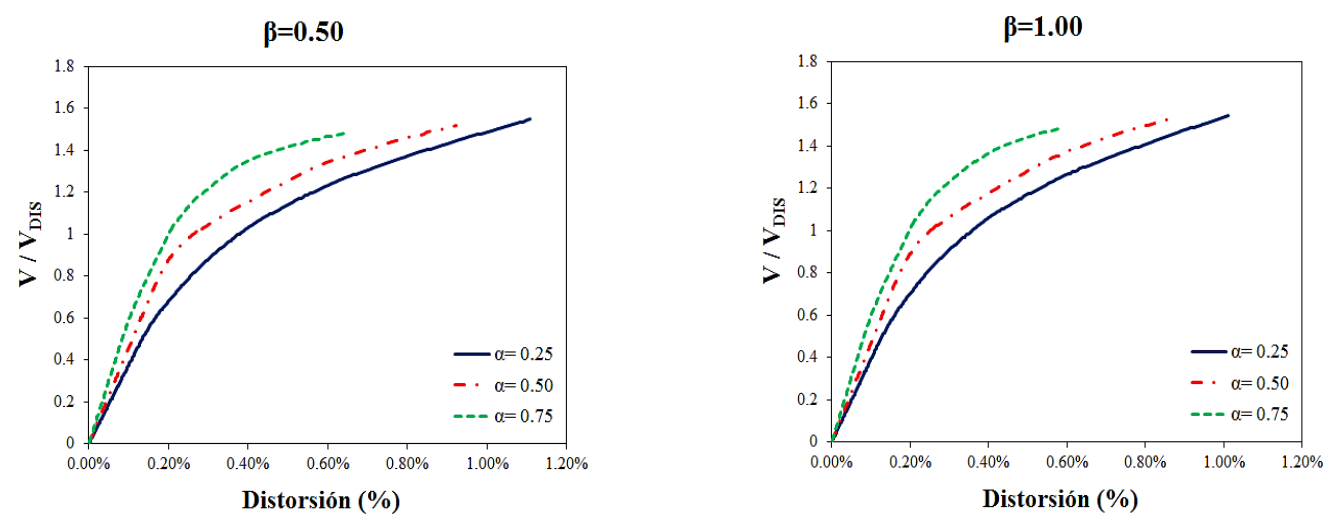

Figura 23. Curvas de cortante basal normalizado-distorsión global de azotea, modelos de 25 niveles con detallado por confinamiento dúctil

En las figuras 22 y 23 se exhiben tres curvas diferentes para comparar el efecto de variar la relación de rigidez entre marco y el sistema contraviento-disipador $(\alpha)$. De forma general, se observa que conforme el valor " $\alpha$ " es menor, la ductilidad desarrollada por los modelos aumenta. De manera contraria, cuando la magnitud de " $\alpha$ " tiende a aumentar, se aprecia que los modelos de 15 niveles presentan menor nivel de sobrerresistencia (figura 22). Esta particularidad se fundamenta en el proceso de diseño de los contravientos; dado que deberán permanecer elásticos durante todo el evento, se sobrediseñan para que no desarrollen pandeo alguno y, conforme el marco aporta mayor rigidez, las secciones de los contravientos tienden a ser menores y, con ello, la sobrerresistencia que pueden desarrollar de manera local se ve reflejada en los parámetros globales.

También se aprecian diferencias entre las rigideces elásticas para los modelos conforme varía el parámetro " $\alpha$ "; generalmente conforme $\alpha$ aumenta, la rigidez elástica disminuye (figura 22). Esta tendencia se observa para modelos de cinco, 10 y 15 niveles. En otras palabras, conforme el marco aporta menor rigidez al sistema, la estructura completa tiende a ser más rígida. Lo anterior se ve reflejado en que las secciones transversales tipo de los contravientos son más grandes, y transmiten mayor carga axial a las columnas y, por ende, éstas tienden a ser más robustas también (Tablas 8 y 10), con lo que el sistema 
global es más rígido cuando " $\alpha=0.25$ ", dado que las secciones tipo se mantienen en varios niveles (Figura $1)$.

A medida que los modelos son más esbeltos (20 y 25 niveles, figura 23) se aprecia que a mayor valor de " $\alpha$ ", la estructura completa es más rígida también. El efecto de axialización en columnas, provocado por la transferencia de carga axial de los contravientos a los elementos del marco, provoca que las secciones transversales de columnas y de vigas resulten más robustas para poder tomar las acciones de diseño. Por ello, se presentan en las tablas 8 a 11, las secciones transversales de los elementos de concreto, con su respectivo refuerzo longitudinal, y las secciones de acero utilizados en contravientos para los modelos de 15 y 25 niveles a diferente balance de rigidez " $\alpha$ ".

Tabla 8. Detallado de armado longitudinal para modelo 15 niveles, $\alpha=0.25, \beta=0.50$, unidades: $\mathrm{cm}$

\begin{tabular}{|c|c|c|c|c|c|}
\hline \multirow{4}{*}{ Vigas } & Entrepisos & b & $\mathbf{h}$ & Lecho Sup. & Lecho Inf. \\
\hline & V 1-5 & 60 & 90 & 11 Vs No. 6 & 10 Vs No. 6 \\
\hline & V 6-10 & 50 & 75 & 11 Vs No. 6 & 10 Vs No. 6 \\
\hline & V 11-15 & 40 & 65 & 7 Vs No. 6 & 7 Vs No. 6 \\
\hline \multirow{4}{*}{ Columnas } & Entrepisos & b & h & Rfzo. Longitudinal & Cuantía \\
\hline & C $1-5$ & 90 & 90 & 28 Vs No. 10 & $2.74 \%$ \\
\hline & C 6-10 & 80 & 80 & 28 Vs No. 8 & $2.22 \%$ \\
\hline & C $11-15$ & 70 & 70 & 20 Vs No. 6 & $1.16 \%$ \\
\hline \multirow{6}{*}{ Contravientos } & Entrepisos & b & h & $\mathbf{t}$ & \\
\hline & CV 1-3 & 55.88 & 55.88 & 7.62 & \\
\hline & CV 4-6 & 48.26 & 48.26 & 6.99 & \\
\hline & CV 7-10 & 40.64 & 40.64 & 5.72 & \\
\hline & CV 10-12 & 35.56 & 35.56 & 3.81 & \\
\hline & CV $13-15$ & 30.48 & 30.48 & 3.18 & \\
\hline
\end{tabular}

Tabla 9. Detallado de armado longitudinal para modelo 15 niveles, $\alpha=0.75, \beta=0.50$, unidades: $\mathrm{cm}$

\begin{tabular}{|c|c|c|c|c|c|}
\hline \multirow{4}{*}{ Vigas } & Entrepisos & $\mathbf{b}$ & $\mathbf{h}$ & Lecho Sup. & Lecho Inf. \\
\hline & V 1-5 & 60 & 90 & 9 Vs No. $8+2$ Vs No. 8 & 9 Vs No. $8+2$ Vs No. 8 \\
\hline & V 6-10 & 50 & 75 & 8 Vs No. $8+2$ Vs No. 8 & 8 Vs No. $8+2$ Vs No. 8 \\
\hline & V 11-15 & 40 & 65 & 6 Vs No. $8+2$ Vs No. 6 & 6 Vs No. $8+2$ Vs No. 6 \\
\hline \multirow{4}{*}{ Columnas } & Entrepisos & $\mathbf{b}$ & $\mathbf{h}$ & Rfzo. Longitudinal & Cuantía \\
\hline & C $1-5$ & 85 & 85 & 32 Vs No. 10 & $3.51 \%$ \\
\hline & C 6-10 & 75 & 75 & 28 Vs No. 10 & $3.94 \%$ \\
\hline & C $11-15$ & 65 & 65 & 20 Vs No. 8 & $2.40 \%$ \\
\hline \multirow{6}{*}{ Contravientos } & Entrepisos & $\mathbf{b}$ & $\mathbf{h}$ & $\mathbf{t}$ & \\
\hline & CV 1-3 & 40.64 & 40.64 & 1.91 & \\
\hline & CV 4-6 & 35.56 & 35.56 & 1.91 & \\
\hline & CV 7-10 & 25.4 & 25.4 & 1.27 & \\
\hline & CV 10-12 & 20.32 & 20.32 & 0.95 & \\
\hline & CV 13-15 & 15.24 & 15.24 & 0.95 & \\
\hline
\end{tabular}


Tabla 10. Detallado de armado longitudinal para modelo 25 niveles, $\alpha=0.25, \beta=0.50$, unidades: $\mathrm{cm}$

\begin{tabular}{|c|c|c|c|c|c|}
\hline \multirow{6}{*}{ Vigas } & Entrepisos & $\mathbf{b}$ & $\mathbf{h}$ & Lecho Sup. & Lecho Inf. \\
\hline & V 1-5 & 75 & 110 & 12 Vs No. 8 & 10 Vs No. 8 \\
\hline & V 6-10 & 65 & 100 & 11 Vs No. 8 & 9 Vs No. $8+2$ Vs No. 6 \\
\hline & V 11-15 & 55 & 85 & 9 Vs No. $8+2$ Vs No. 6 & 8 Vs No. $8+3$ Vs No. 6 \\
\hline & V 16-20 & 45 & 70 & 7 Vs No. $8+2$ Vs No. 6 & 6 Vs No. $8+3$ Vs No. 6 \\
\hline & V 21-25 & 35 & 55 & 5 Vs No. 8 & 3 Vs No. $8+3$ Vs No. 6 \\
\hline \multirow{6}{*}{ Columnas } & Entrepisos & b & $\mathbf{h}$ & Rfzo. Longitudinal & Cuantía \\
\hline & C $1-5$ & 150 & 150 & 92 Vs No. 10 & $3.24 \%$ \\
\hline & C 6-10 & 135 & 135 & 84 Vs No. 10 & $3.65 \%$ \\
\hline & C $11-15$ & 120 & 120 & 68 Vs No. 10 & $3.74 \%$ \\
\hline & C $16-20$ & 105 & 105 & 44 Vs No. 10 & $3.16 \%$ \\
\hline & C $21-25$ & 90 & 90 & 20 Vs No. 10 & $1.95 \%$ \\
\hline \multirow{7}{*}{ Contravientos } & Entrepisos & b & $\mathbf{h}$ & $\mathbf{t}$ & \\
\hline & CV 1-4 & 71.12 & 71.12 & 25.72 & \\
\hline & CV 5-8 & 63.5 & 63.5 & 19.05 & \\
\hline & CV 9-12 & 50.8 & 50.8 & 17.78 & \\
\hline & CV 13-17 & 40.64 & 40.64 & 12.7 & \\
\hline & CV 18-21 & 30.48 & 30.48 & 7.62 & \\
\hline & CV 22-25 & 30.48 & 30.48 & 2.54 & \\
\hline
\end{tabular}

Tabla 11. Detallado de armado longitudinal para modelo 25 niveles, $\alpha=0.75, \beta=0.50$, unidades: $\mathrm{cm}$

\begin{tabular}{|c|c|c|c|c|c|}
\hline \multirow{6}{*}{ Vigas } & Entrepisos & b & $\mathbf{h}$ & Lecho Sup. & Lecho Inf. \\
\hline & V 1-5 & 85 & 125 & 15 Vs No. $8+7$ Vs No. 6 & 13 Vs No. $8+9$ Vs No. 6 \\
\hline & V 6-10 & 75 & 115 & 13 Vs No. $8+6$ Vs No. 8 & 13 Vs No. $8+5$ Vs No. 8 \\
\hline & V 11-15 & 70 & 105 & 12 Vs No. $8+8$ Vs No. 6 & 11 Vs No. $8+9$ Vs No. 6 \\
\hline & V 16-20 & 60 & 90 & 10 Vs No. $8+6$ Vs No. 6 & 9 Vs No. $8+7$ Vs No. 6 \\
\hline & V 21-25 & 50 & 75 & 7 Vs No. $8+3$ Vs No. 6 & 6 Vs No. $8+4$ Vs No. 6 \\
\hline \multirow{6}{*}{ Columnas } & Entrepisos & $\mathbf{b}$ & $\mathbf{h}$ & Rfzo. Longitudinal & Cuantía \\
\hline & C $1-5$ & 145 & 145 & 92 Vs No. 10 & $3.46 \%$ \\
\hline & C 6-10 & 130 & 130 & 84 Vs No. 10 & $3.94 \%$ \\
\hline & C $11-15$ & 120 & 120 & 68 Vs No. 10 & $3.74 \%$ \\
\hline & C $16-20$ & 105 & 105 & 44 Vs No. 10 & $3.16 \%$ \\
\hline & C $21-25$ & 90 & 90 & 24 Vs No. 10 & $2.35 \%$ \\
\hline \multirow{7}{*}{ Contravientos } & Entrepisos & $\mathbf{b}$ & $\mathbf{h}$ & $\mathbf{t}$ & \\
\hline & CV 1-4 & 45.72 & 45.72 & 5.72 & \\
\hline & CV 5-8 & 40.64 & 40.64 & 4.45 & \\
\hline & CV 9-12 & 35.56 & 35.56 & 4.13 & \\
\hline & CV 13-17 & 30.48 & 30.48 & 3.81 & \\
\hline & CV 18-21 & 25.4 & 25.4 & 2.54 & \\
\hline & CV 22-25 & 20.32 & 20.32 & 1.59 & \\
\hline
\end{tabular}

Para los modelos de 15 niveles, las secciones geométricas utilizadas para formar el marco son similares para ambos balances de " $\alpha$ ", con variación en los refuerzos longitudinales de vigas y columnas para satisfacer las demandas impuestas en el proceso de diseño, teniendo que proveer mayor cantidad de acero de refuerzo en $\alpha=0.75$ (Tabla 9). Para los modelos de 25 niveles, el refuerzo longitudinal empleado en los elementos de concreto fue cercano al máximo permisible para ambos balances de " $\alpha$ ", pero se decidió utilizar diferentes secciones transversales en vigas, siendo más robustas para el balance de rigidez 
" $\alpha=0.75 "$ (Tabla 11). Con la anterior descripción, se infiere que para estructuras con disipadores de energía, diseñados con una metodología de balances de rigidez entre sus sistemas, la variación de las secciones de vigas y columnas, para diferentes balances de " $\alpha$ ", influyen en gran medida en la rigidez global del sistema completo en estructuras esbeltas.

En la figura 24 se observa que el sistema estructural completo desarrolla menor ductilidad conforme el valor de " $\beta$ " es mayor, bajo las mismas condiciones de " $\alpha$ ". Se aprecian también diferencias en la rigidez elástica, debido a cambios en las secciones transversales de los contravientos pero sin variación en las secciones de vigas y columnas; y en la rigidez post-fluencia, consecuencia de las características del disipador obtenidas en su diseño. También es importante mencionar que conforme aumenta el número de niveles, la distorsión global desarrollada por los modelos es mayor, y su sobrerresistencia tiende a disminuir.
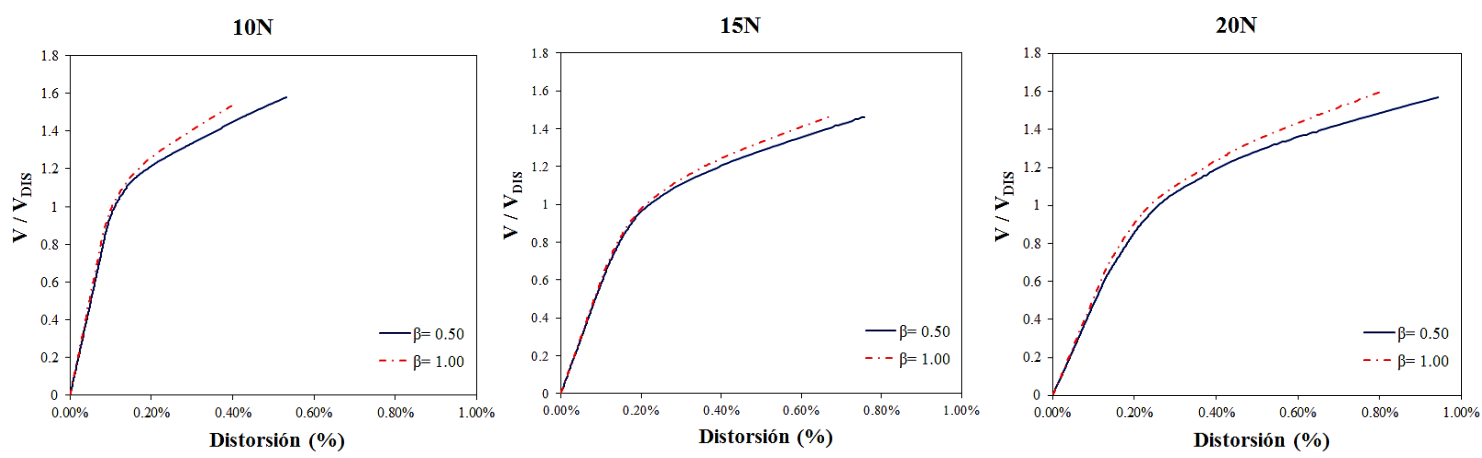

Figura 24. Comparativa curvas de cortante basal normalizado-distorsión global de azotea, $\alpha=0.25$ con detallado por confinamiento dúctil

La mejor manera de comparar el parámetro de confinamiento, acorde con los niveles de detallado dúctil que se proporcionaron a los elementos de concreto reforzado, es por medio del mapeo de fluencias inelásticas entre los marcos sin detallado dúctil y los correspondientes con detallado por confinamiento dúctil. En la figura 25 se presenta de manera ilustrativa la escala de colores asignada para los elementos de concreto. El punto en color blanco, representa el punto a partir del cual el elemento empieza a incursionar en el intervalo inelástico; el punto en color negro, representa la curvatura última que el elemento de concreto reforzado es capaz de soportar, los puntos amarillo, naranja y rojo, son puntos intermedios entre el punto de fluencia y el punto último que soporta la sección.

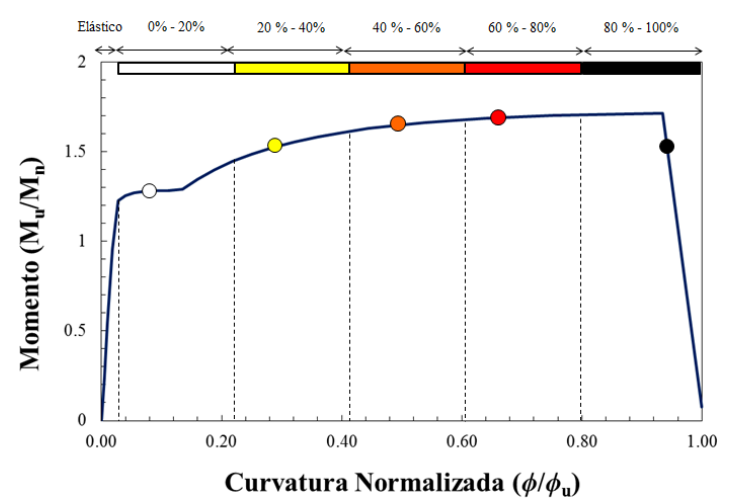

Figura 25. Escala de colores para el mapeo de fluencias inelásticas de vigas y columnas 
En las figuras 26 y 27 se presenta una comparativa para los modelos de 15 y 25 niveles respectivamente (con y sin confinamiento dúctil), a partir de los mapeos de fluencia correspondientes a la ductilidad objetivo de los disipadores $\mu_{d}$, y se observan las diferencias de proveer a las estructuras con requisitos mínimos de detallado por confinamiento dúctil. Se aprecia que el uso de los requisitos antes mencionados proporciona una mayor capacidad de rotación elástica a vigas y columnas. Esto se observa de manera esquemática en el cambio de códigos de color en algunas vigas, pasando de amarillo a blanco (daño incipiente), y en el caso de las columnas se aprecia que las fluencias logran desaparecer y éstas permanecen elásticas, incluso para el modelo más esbelto.

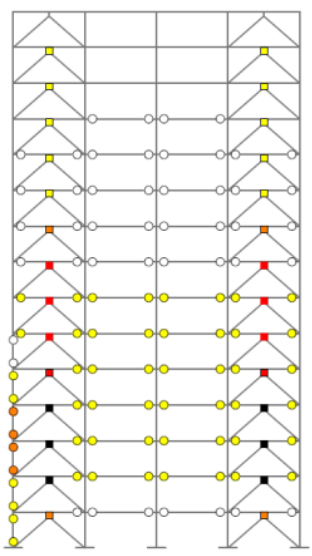

a) Detallado no dúctil

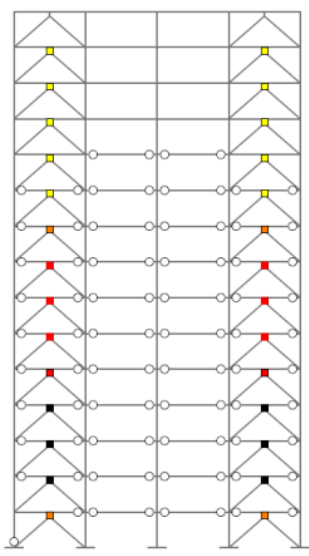

b) Detallado dúctil

Figura 26. Comparativa de mapeos de fluencia a la ductilidad objetivo de los disipadores $\mu_{\mathrm{d}}$ para modelos de 15 niveles $\operatorname{con} \alpha=0.25, \beta=0.50$

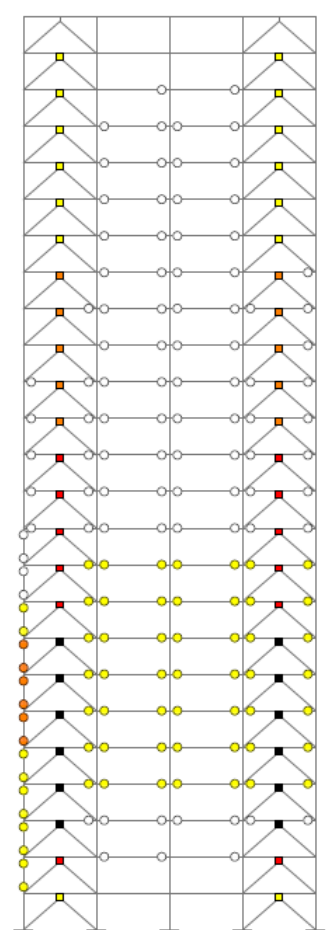

a) Detallado no dúctil

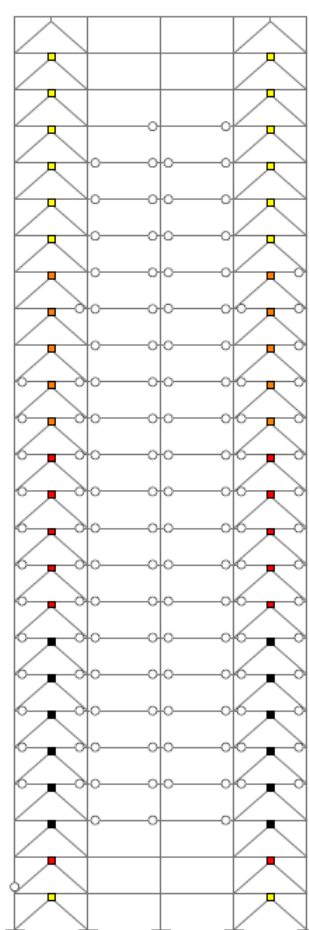

b) Detallado dúctil

Figura 27. Comparativa de mapeos de fluencia a la ductilidad objetivo de los disipadores $\mu_{\mathrm{d}}$ para modelos de 25 niveles $\operatorname{con} \alpha=0.50, \beta=0.50$ 
En la figura 28 se presenta el mapeo de fluencias a la ductilidad objetivo de los disipadores $\mu_{d}$, para los modelos de 20 niveles con una rigidez de los disipadores equivalente a la mitad de rigidez del sistema de contraviento $(\beta=0.50)$. A diferencia de los modelos de 5, 10 y 15 niveles, donde el balance óptimo era " $\alpha=0.25$ " (comportamiento representativo de la figura 26), para los modelos más esbeltos estudiados, en dicho balance se observa la formación de fluencias en ambos extremos de las columnas de la periferia. Además, el nivel de daño asociado a la fluencia de vigas ya no es incipiente, sino por el contrario, se prevé un daño notorio, por lo que en un evento post-sísmico requerirá de reparación, y con esto, el incumplimiento de la filosofía de diseño para estructuras con disipadores. Además, se observa que conforme el balance " $\alpha$ " aumenta, la magnitud de las rotaciones que se presentan en los modelos es menor. Esto es consecuencia del diseño de los elementos del marco, que conforme más rigidez toma este sistema, vigas y columnas se diseñan para que resistan más cortante del sistema global.

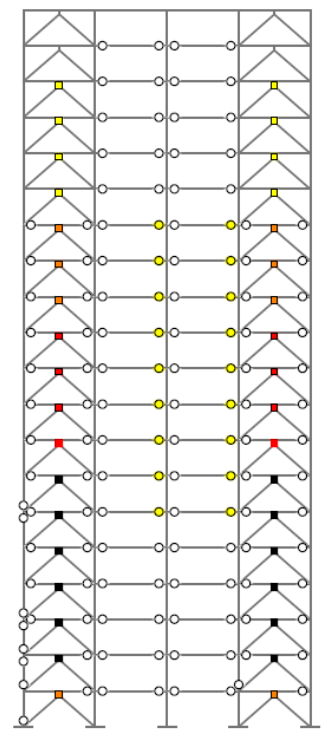

a) $\alpha=0.25$

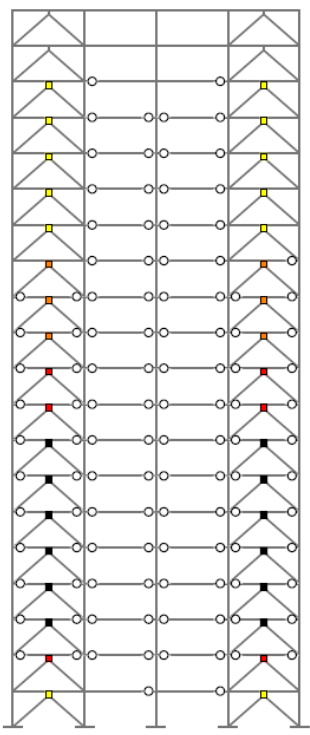

b) $\alpha=0.50$

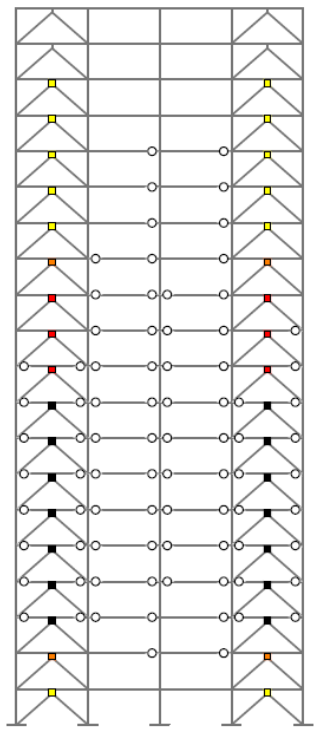

c) $\alpha=0.75$

Figura 28. Comparativa de mapeos de fluencia a la ductilidad objetivo de los disipadores $\mu_{\mathrm{d}}$ para modelos de 20 niveles con detallado por confinamiento dúctil con $\beta=0.50$

De manera similar que para el modelo de 20 niveles, en la figura 29 se presenta el mapeo de fluencias para el modelo de 25 niveles con diferentes balances de rigidez " $\alpha$ ". Se observa que para el balance " $\alpha=0.25$ " existen rotaciones no incipientes en vigas, así como fluencias en ambos extremos de las columnas en los entrepisos donde los disipadores alcanzan su ductilidad máxima. Observando el mapeo de rotaciones para los balances de " $\alpha=0.50$ " y " $\alpha=0.75$ ", se aprecia que en el último, hay mayor cantidad de disipadores que alcanzan la ductilidad objetivo, pero si se compara la ductilidad global del sistema que puede desarrollar, el del balance " $\alpha=0.75$ " es menor al del balance de " $\alpha=0.50$ ", lo que se detallará más adelante.

Con base en las observaciones señaladas a partir del análisis de los mapeos de fluencia, se concluye que conforme los modelos son más esbeltos, la rigidez necesaria que debe aportar el sistema del marco al sistema global $(\alpha)$ debe ser mayor para mitigar el efecto de la axialización de las columnas y un daño perceptible en las vigas, tal y como se aprecia de las figuras 28 y 29 para el balance de " $\alpha=0.25$ ". 


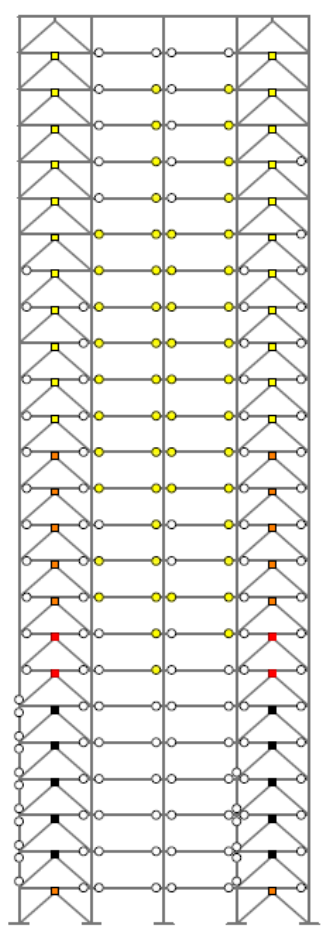

a) $\alpha=0.25$

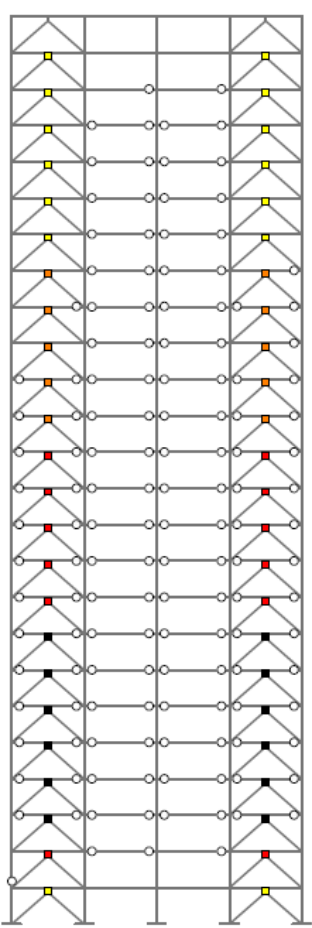

b) $\alpha=0.50$

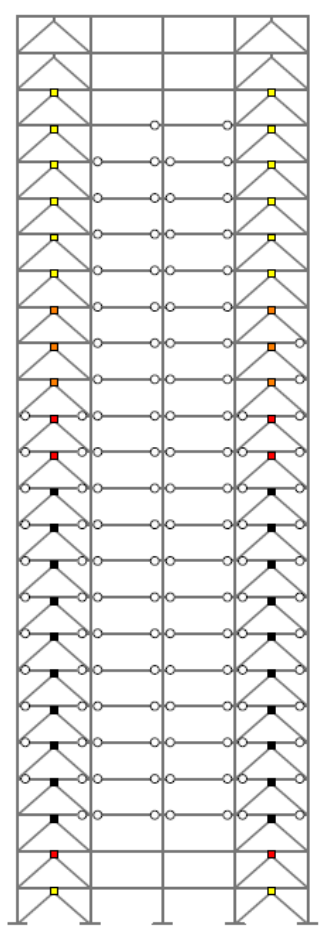

c) $\alpha=0.75$

Figura 29. Comparativa de mapeos de fluencia a la ductilidad objetivo de los disipadores $\mu_{\mathrm{d}}$ para modelos de 25 niveles con detallado por confinamiento dúctil con $\beta=0.50$

\section{BALANCES DE RIGIDEZ “ÓPTIMOS”}

En trabajos previos (Nangullasmú 2011, Tena y Nangullasmú 2013, Tena-Colunga y NangullasmúHernández 2015), se observó que diseñar a los elementos de concreto reforzado con requisitos de marcos no dúctiles (NTCC 2004), fomenta la participación no lineal en vigas y columnas, por lo que el sistema estructural global no se comportará con un desempeño acorde con la filosofía de diseño de estructuras con disipadores de energía. Los balances "óptimos" que se obtuvieron para elementos de concreto sin detallados de confinamiento dúctil se indican en la tabla 10, donde los únicos modelos que se comportaron de manera satisfactoria fueron los más pequeños del estudio (marcos de cinco niveles). Los otros modelos tuvieron un desempeño aceptable, pero no del todo favorable, debido a que la participación no lineal no es exclusiva de los disipadores de energía.

En las tablas 10 y 11 se presenta el resumen con los parámetros recomendados asociados a los balances de rigidez entre los sistemas. Se usan tres colores para identificar el nivel de daño que se puede presentar si se elige alguna de las combinaciones mostradas en las tablas. El color blanco representa que las columnas permanecen elásticas cuando los disipadores desarrollan su ductilidad objetivo, mientras que las vigas pueden permanecer elásticas o tener una participación incipiente. El color amarillo, representa una participación no lineal incipiente en vigas y columnas. El color naranja, representa que vigas y columnas tienen una participación de mediana a importante en la no linealidad del sistema global.

Si se compara la información provista en las tablas 10 y 11, se aprecia que con el uso del detallado dúctil en vigas y columnas amplía el número de combinaciones posibles donde el sistema del marco tiene menor participación no lineal dentro del sistema estructural global. Para los modelos esbeltos con un 
balance de " $\alpha=0.25$ ", proporciona mayor ductilidad al sistema global, pero repercute en la participación no lineal de algunas columnas.

Tabla 10. Parámetros estructurales recomendados para marcos de concreto no dúctiles con disipadores de energía

\begin{tabular}{|c|c|c|c|c|c|c|}
\hline Niveles & $\alpha$ & $K_{2}$ & & $\beta$ & & $\mu$ \\
\hline \multirow{3}{*}{5} & \multirow{3}{*}{0.25} & 0.00 & 0.5 & - & 1.0 & $10-6$ \\
\hline & & 0.03 & 0.5 & - & 1.0 & $10-8$ \\
\hline & & 0.05 & 0.5 & - & 1.0 & $10-8$ \\
\hline \multirow{3}{*}{10} & \multirow{3}{*}{0.25} & 0.00 & 0.5 & - & 1.0 & $8-6$ \\
\hline & & 0.03 & 0.5 & - & 1.0 & $12-8$ \\
\hline & & 0.05 & 0.5 & - & 1.0 & $12-8$ \\
\hline \multirow{3}{*}{15} & \multirow{3}{*}{0.25} & 0.00 & 0.5 & - & 1.0 & $<6$ \\
\hline & & 0.03 & 0.5 & - & 1.0 & $12-8$ \\
\hline & & 0.05 & 0.5 & - & 1.0 & $12-8$ \\
\hline \multirow{3}{*}{20} & \multirow{3}{*}{0.50} & 0.00 & 0.5 & - & 1.0 & $10-8$ \\
\hline & & 0.03 & 0.5 & - & 1.0 & $12-8$ \\
\hline & & 0.05 & 0.5 & - & 1.0 & $12-8$ \\
\hline \multirow{3}{*}{25} & \multirow{3}{*}{0.50} & 0.00 & 0.5 & - & 1.0 & $10-6$ \\
\hline & & 0.03 & 0.5 & - & 1.0 & $12-8$ \\
\hline & & 0.05 & 0.5 & - & 1.0 & $12-8$ \\
\hline
\end{tabular}

Tabla 11. Parámetros estructurales recomendados para marcos de concreto no dúctiles con detallado por confinamiento dúctil en vigas y columnas y con disipadores de energía

\begin{tabular}{cccccc}
\hline Niveles & $\boldsymbol{\alpha}$ & $\boldsymbol{K}_{\mathbf{2}}$ & & $\boldsymbol{\beta}$ & $\boldsymbol{\mu}$ \\
\hline \multirow{3}{*}{5} & 0.25 & & $0.5-$ & 1.0 & $10-8$ \\
& 0.50 & 0.05 & $0.5-$ & 1.0 & $10-6$ \\
& 0.75 & & $0.5-$ & 1.0 & $12-8$ \\
\hline \multirow{2}{*}{10} & 0.25 & & $0.5-$ & 1.0 & $12-8$ \\
& 0.50 & 0.05 & $0.5-$ & 1.0 & $10-8$ \\
& 0.75 & & $0.5-$ & 1.0 & $10-8$ \\
\hline \multirow{2}{*}{15} & 0.25 & & $0.5-$ & 1.0 & $12-8$ \\
& 0.50 & 0.05 & $0.5-$ & 1.0 & $12-8$ \\
& 0.75 & & $0.5-$ & 1.0 & $12-8$ \\
\hline \multirow{2}{*}{20} & 0.25 & & $0.5-$ & 1.0 & $12-8$ \\
& 0.50 & 0.05 & $0.5-$ & 1.0 & $12-8$ \\
& 0.75 & & $0.5-$ & 1.0 & $12-8$ \\
\hline \multirow{2}{*}{25} & 0.25 & & $0.5-$ & 1.0 & $12-6$ \\
& 0.50 & 0.05 & $0.5-$ & 1.0 & $12-8$ \\
& 0.75 & & $0.5-$ & 1.0 & $12-10$ \\
\hline
\end{tabular}

En la figura 30 se presenta el mapeo de fluencias de los modelos que presentaron un mejor desempeño estructural cuando se les provee de un confinamiento dúctil en vigas y columnas, tomando en cuenta:

a) La ductilidad que desarrollan los disipadores en cada uno de los niveles $\left(\mu_{d}\right)$

b) La ductilidad global desarrollado por el sistema estructural global $(Q)$

c) La magnitud de las fluencias de los elementos de concreto reforzado 
Se aprecia que para los balances presentados en esta figura, las columnas permanecen elásticas y las vigas con una participación no lineal incipiente o apenas perceptible. Si se compara la figura 30 (marcos con detallado para confinamiento dúctil) con la figura 7 (marcos con detallado para confinamiento no dúctil), se aprecia que el adicionar requisitos mínimos de detallado por confinamiento mejoran considerablemente el desempeño estructural de los marcos de concreto con disipadores de energía por histéresis del material. Es importante recalcar que la adición de estos requisitos mínimos al proceso de diseño (Ap. 7.2.3 y 7.3.4), es una opción que algunos despachos de cálculo estructural mexicanos utilizan como alternativa factible para no seguir de manera rigurosa todas las pautas que se especifican dentro del código para marcos dúctiles de concreto reforzado; es decir, sin tener que satisfacer todos los requerimientos de la sección 7 de las Normas Técnicas Complementarias de Estructuras de Concreto (Tena-Colunga et al. 2008).

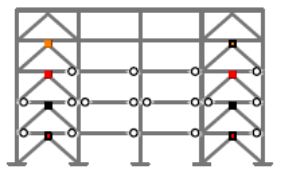

a) $\alpha=0.25$

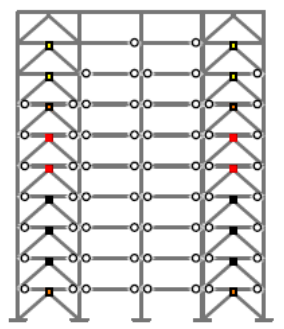

b) $\alpha=0.25$

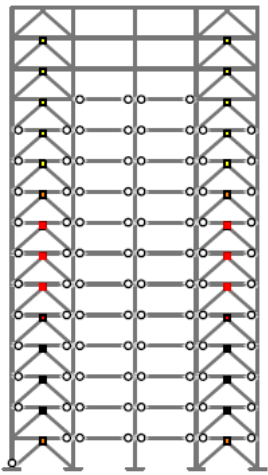

c) $\alpha=0.25$

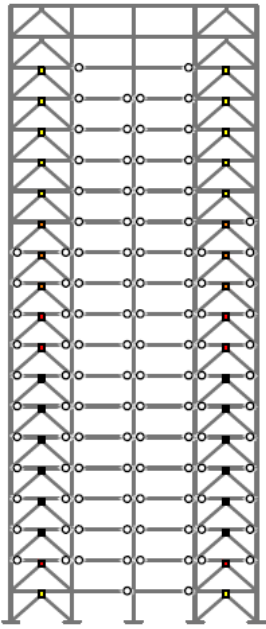

d) $\alpha=0.50$

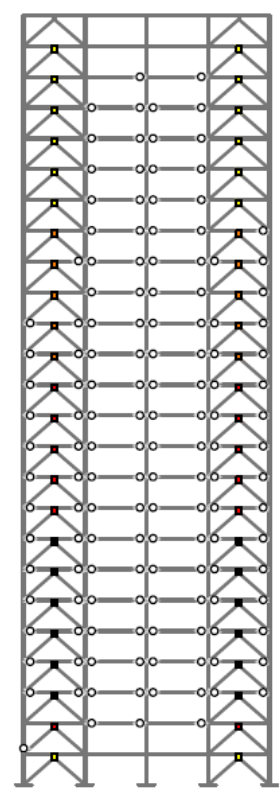

e) $\alpha=0.50$

Figura 30. Mapeos de fluencia a la ductilidad objetivo de los disipadores $\mu_{\mathrm{d}}$ para los modelos con detallado por confinamiento dúctil para balances óptimos de rigidez $\alpha$ cuando $\beta=0.50$

\section{PARÁMETROS PROPUESTOS}

Con base en el universo de modelos evaluados, y para el futuro diseño de modelos más complejos, a continuación se proponen algunos parámetros de diseño conforme a las Normas Técnicas Complementarias para diseño por Sismo (NTCS-04). Es importante mencionar que para modelos de 5, 10 y 15 niveles, el balance "óptimo" es $\alpha=0.25$, mientras que para modelos de 20 y 25 niveles, el balance "óptimo" es $\alpha=0.50$. Por cuestiones prácticas y de rápida visualización, en cada una de las gráficas siguientes se encuentran enmarcados con un círculo color rojo los balances "óptimos" antes mencionados, graficando en el eje horizontal la relación de esbeltez de cada uno de los modelos estudiados y en el eje vertical la magnitud del factor de comportamiento sísmico respectivo.

Para los valores de reducción por sobrerresistencia, se observa de la figura 31 que dicho valor es mayor para los modelos de menor altura, y que conforme va aumentando el número de niveles analizados, éste valor tiende a 1.5. Si se observan los puntos enmarcados en círculos (balances de rigidez "óptimos"), se distingue que proponer un valor de $\mathbf{R}=\mathbf{1 . 5}$ es un valor promedio práctico para los modelos que presentaban un desempeño aceptable. 


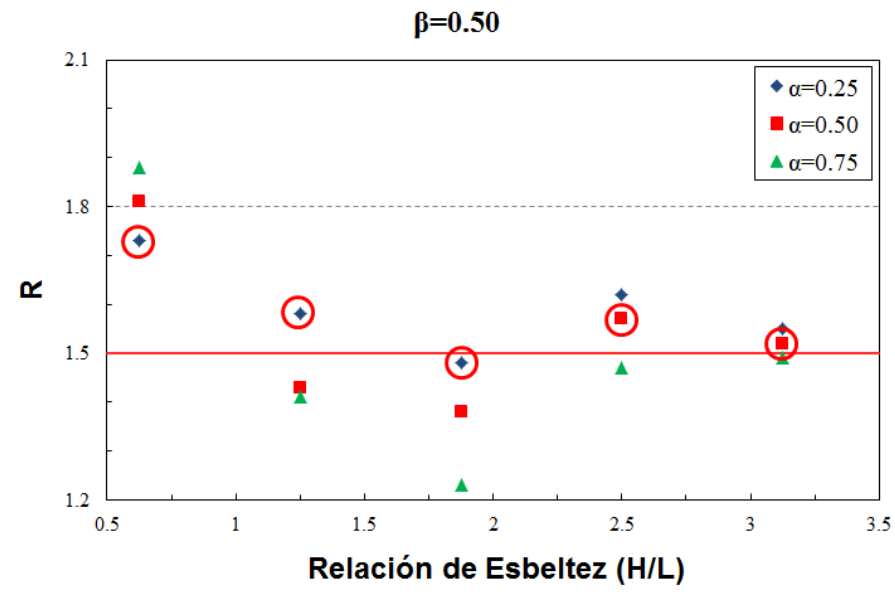

Figura 31. Factor de reducción por sobrerresistencia, R

En la figura 32 se observan los valores del factor de comportamiento sísmico $Q$ (o de reducción por ductilidad) que desarrolló el sistema global en los marcos evaluados. Se aprecia que conforme aumenta el número de niveles de los modelos, la ductilidad global desarrollada tiende a disminuir. Además, en esta gráfica se visualiza que usar un balance $\alpha=0.75$ tiende a desarrollar una ductilidad $Q$ mucho menor que para los otros balances de rigidez propuestos $(Q \approx 3.0)$. Si se observan los puntos enmarcados en círculos

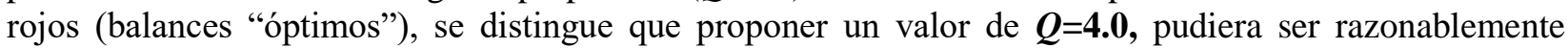
práctico para el diseño estructural de edificios con disipadores histeréticos de energía que cumplan con las condiciones de regularidad establecidas en las NTCS-04 (relación de esbeltez $H / L \leq 2.5$ ).

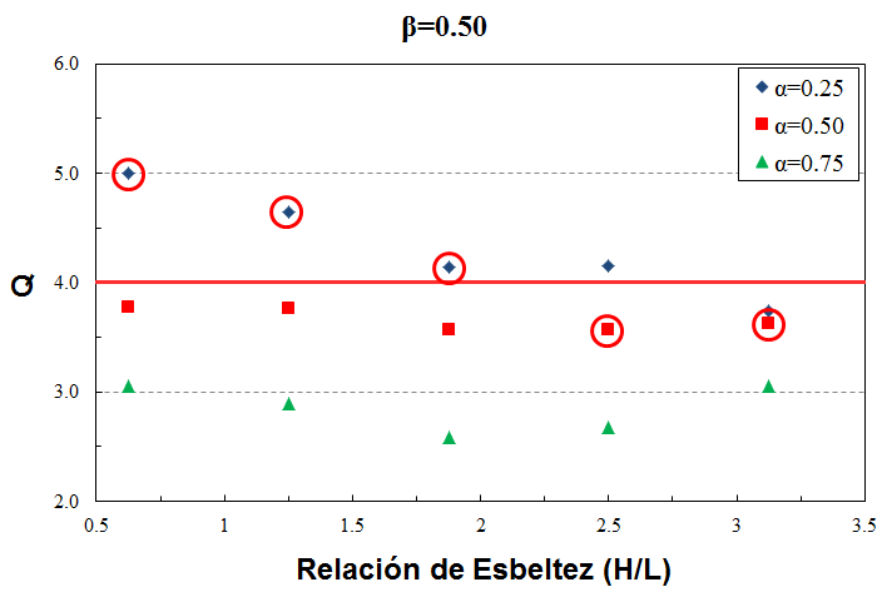

Figura 32. Factor de comportamiento sísmico $Q$

\section{CONCLUSIONES}

En el presente trabajo se estudió la importancia de emplear requisitos mínimos de detallado dúctil por confinamiento como complemento adicional en el diseño de estructuras con base en marcos no dúctiles de concreto reforzado con disipadores histeréticos de energía montados en contraventeo chevrón, diseñados conforme a los lineamientos de las NTCC-04. La finalidad del estudio es mejorar el desempeño estructural global de este tipo de estructuras acorde con la filosofía de diseño de marcos a momento provistos con control pasivo de la respuesta sísmica. 
De los resultados detallados antes presentados, se observó que el adicionar con requisitos mínimos de detallado dúctil a los elementos de concreto reforzado mejoró notablemente el comportamiento del sistema global, permitiendo que los elementos del marco tengan una participación no lineal casi imperceptible en la gran mayoría de modelos estudiados. Con la elección de balances de rigidez "óptimos", se logró que las columnas se comporten de manera elástica, a pesar que las vigas, para todos los modelos evaluados, tienen una participación incipiente en la no linealidad del sistema.

Con la comparación de resultados entre el usar y no usar detallados dúctiles por confinamiento, se ha ampliado el espectro de balances de rigidez "óptimos" que un ingeniero de la práctica pudiera usar para diseñar estructuras de este tipo, sin que el sistema del marco sufra daño que provoque el paro de actividades del inmueble. Se distingue la importancia que tiene en el desempeño estructural, la separación y el número de ramas de estribos que se les proporciona a los elementos de concreto. El hacer más estrecha dicha separación en la zona de los extremos del elemento, permite mayor capacidad de deformación con menor daño en vigas y columnas.

Se observó que conforme los modelos analizados son más esbeltos, el aporte de rigidez que debe proporcionar el sistema del marco $(\alpha)$ debe ser mayor para evitar daño notorio en los elementos de concreto reforzado. La importancia del parámetro " $\beta$ " (balance de rigidez entre contraviento y disipador) reside en la ductilidad desarrollada por los disipadores y su impacto en las demandas inelásticas en vigas y columnas. En general, mientras el balance " $\beta$ " disminuye, los disipadores tienden a desarrollar mayor magnitud de ductilidad local, pero esto también lidera a mayores demandas inelásticas en los elementos del marco, principalmente en columnas.

Se han propuesto factores de comportamiento sísmico acorde con el reglamento del Distrito Federal vigente, para un proceso de diseño conforme a lo que actualmente se conoce y se utiliza en la práctica ingenieril mexicana. Se distingue que el factor de comportamiento sísmico $Q$ tiende a disminuir conforme el número de niveles aumenta, y que proponer un valor $\boldsymbol{Q}=\mathbf{4 . 0}$ es razonable, desde un punto de vista práctico, para estructuras que cumplen la condición de regularidad por esbeltez $(H / L \leq 2.5)$. Además, se observa que los valores de sobrerresistencia tienden a ser mayores conforme la rigidez que aporta el sistema contraviento-disipador es mayor $(\alpha=0.25)$, con excepción para el modelo más pequeño.

Finalmente, el estudio demuestra que el emplear de manera voluntaria los requisitos de confinamiento dúctil no impacta significativamente a los costos de construcción. Tomando exclusivamente los costos directos asociados al marco de concreto reforzado (vigas y columnas), para todos los modelos estudiados con sus distintos balances de rigideces $\alpha$ y $\beta$, se obtuvo que emplear un detallado por confinamiento dúctil está asociado a un incremento en costos entre el $6 \%$ y el $12 \%$ (en promedio 10\%), siendo los modelos más esbeltos donde se aprecia la mayor diferencia en costos. Por lo tanto, el impacto económico real es mucho menor, dado que no se incluyeron los costos de contravientos y disipadores (que son los mismos en los modelos sin confinamiento dúctil y con confinamiento dúctil), y los de la cimentación, que muy seguramente sería la misma también, dado que el incremento del peso total del marco sería poco significativo. Los costos por mano de obra no deben incrementarse de manera importante, pues sólo involucran el habilitado y colocación de estribos adicionales en vigas y columnas antes del colado.

\section{AGRADECIMIENTOS}

El primer autor agradece la beca otorgada por Conacyt, que le permitió involucrarse en este estudio en el desarrollo de su tesis doctoral. 


\section{REFERENCIAS}

Calado, L, J Proença, M Espinha y C Castiglioni (2013), "Hysteretic behaviour of dissipative bolted fuses for earthquake resistant steel frames”, Journal of Constructional Steel Research, Vol. 85, pp. 151-162. DOI: $10.1016 /$ j.jcsr.2013.02.016

Castiglioni, C, A Kanyilmaz y L Calado (2012), "Experimental analysis of seismic resistant composite steel frames with dissipative devices", Journal of Constructional Steel Research, Vol. 76, pp. 1-12. DOI: 10.1016/j.jcsr.2012.03.027

Ciampi, V, V Paolone y M De Angelis (1992), "On the seismic design of dissipative bracings", Memorias, Tenth World Conference on Earthquake Engineering, Madrid, Spain, 4133-4138. ISBN 9054100605

Ciampi, V, M De Angelis y V Paolone (1995), "Design of yielding or friction-based dissipative bracings for seismic protection of buildings", Engineering Structures, Vol. 17, No. 5, pp. 381-391. DOI: $\underline{10.1016 / 0141-0296(95) 00021-X}$

Chan, R y F Albermani (2008), "Experimental study of steel slit damper for passive energy dissipation", Engineering Structures, Vol. 30, pp. 1058-1066. DOI: 10.1016/j.engstruct.2007.07.005

Esteva, L y L Veras (1998), "Criterios de ductilidad y desempeño para el diseño sísmico de estructuras con disipadores histeréticos de energía”, Memorias, V Simposio Nacional de Ingeniería Sísmica, Toluca, septiembre.

Foti, D, L M Bozzo y F López-Almansa (1998), "Numerical efficiency assessment of energy dissipators for seismic protection of buildings", Earthquake Engineering and Structural Dynamics, Vol. 27, pp. 543556. ISSN: 0098-8847

Ghabraie, K, R Chan, X Huang y Y Xie (2010), "Shape optimization of metallic yielding devices for passive mitigation of seismic energy", Engineering Structures, Vol. 32, pp. 2258-2267. DOI: 10.1016/j.engstruct.2010.03.028

Hanson, R D (1993), "Supplemental damping for improved seismic performance", Earthquake Spectra, Vol. 9, No. 3, pp. 319-334. DOI: 10.1193/1.1585719

Hanson, R D y T T Soong (2001), "Seismic design with supplemental energy dissipation devices", Monograph Series MNO-8, Earthquake Engineering Research Institute. ISBN 0943198135

Housner, G W, L A Bergman, T K Caughey, A G Chassiakos, R O Claus, S F Masri, R E Skelton, T T Soong, B F Spencer y T P Yao (1997), "Structural control: past, present, and future", Journal of Engineering Mechanics, ASCE, Vol. 123, No. 9, pp. 897-971. DOI: 10.1061/(ASCE)07339399(1997)123:9(897)

Jara, J, E Miranda y G Ayala (2007), "Parametric study of single-degree-of-freedom systems with energy dissipating devices built on soft soil sites", Engineering Structures, Vol. 29 , pp. 1398-1413. DOI: 10.1016/j.engstruct.2006.08.018

Jara, J (2009), "Seismic response of buildings with energy dissipating systems built in soft soils", Engineering Structures, Vol. 31, pp. 1204-1216. DOI: 10.1016/j.engstruct.2009.01.015

Karavasilis, T L, S Kerawala y E Hale (2012), "Hysteretic model for steel energy dissipation devices and evaluation of a minimal-damage seismic design approach for steel buildings", Journal of Constructional Steel Research, Vol. 70, pp. 358-367. DOI: 10.1016/j.jcsr.2011.10.010

Lin, Y Y, M H Tsai, J S Hwang y K C Chang (2003), "Direct displacement-based design for building with passive energy dissipation systems", Engineering Structures, Vol. 25, pp. 25-37. DOI: $\underline{10.1016 / \mathrm{S} 0141-0296(02) 00099-8}$ 
Mahmoudi, M y M G Abdi (2012), "Evaluating response modification factors of TADAS frames", Journal of Constructional Steel Research, Vol. 71, pp. 162-170. DOI: 10.1016/j.jcsr.2011.10.015

Martínez, E (1994), Comunicación personal con el Ing. Martínez Romero.

Mualla, I y B Belev (2002), "Performance of steel frames with a new friction damper device under earthquake excitation", Engineering Structures, Vol. 24, pp. 365-371. DOI: 10.1016/S0141$\underline{0296(01) 00102-X}$

Nangullasmú, H J (2011), "Propuesta de criterios de diseño sísmico conforme a reglamento para marcos no dúctiles de concreto reforzado con disipadores histeréticos", Tesis de Maestría, Posgrado en Ingeniería Estructural, División de Ciencias Básicas e Ingeniería, Universidad Autónoma Metropolitana Azcapotzalco, México.

NTCC-04 (2004), "Normas Técnicas Complementarias para Diseño de Estructuras de Concreto", Gaceta Oficial del Distrito Federal, Tomo II, No. 103-BIS, octubre.

NTCM-04 (2004), "Normas Técnicas Complementarias para Diseño de Estructuras Metálicas", Gaceta Oficial del Distrito Federal, Tomo II, No. 103-BIS, octubre.

NTCS-04 (2004), "Normas Técnicas Complementarias para Diseño por Sismo”, Gaceta Oficial del Distrito Federal, Tomo II, No. 103-BIS, octubre.

Oh, S H, Y J Kim y H S Ryu (2009), "Seismic performance of steel structures with slit dampers", Engineering Structures, Vol. 31, pp. 1997-2008. DOI: 10.1016/j.engstruct.2009.03.003

Park, R y T Paulay (1986), Estructuras de Concreto Reforzado, Limusa, México. ISBN-13: 9789681801007

Prakash, V, G H Powell y F C Fillipou (1992), "DRAIN-2DX: base program user guide”, Report No. UBC/SEMM-92/29, Department of Civil Engineering, University of California at Berkeley.

Rai, D, P Annam y T Pradhan (2013), "Seismic testing of steel braced frames with aluminum shear yielding dampers", Engineering Structures, Vol. 46, pp. 737-747. DOI: 10.1016/j.engstruct.2012.08.027

Riobóo, J M (1995), "Estructuras de concreto", Memorias, Simposio Internacional: La ingeniería civil a 10 años de los sismos de 1985, México, D.F, septiembre, pp. 123-127.

Sahoo, D y D Rai (2010), "Seismic strengthening of non-ductile reinforced concrete frames using aluminum shear links as energy-dissipation devices", Engineering Structures, Vol. 32, pp. 3548-3557. DOI: $\underline{10.1016 / j . e n g s t r u c t .2010 .07 .023}$

Shih, M H y W P Sung (2005), "A model for hysteretic behavior of rhombic low yield strength steel added damping and stiffness", Computers and Structures, Vol. 83, pp. 895-908. DOI: $\underline{10.1016 / j . c o m p s t r u c .2004 .11 .012}$

Tehranizadeh, M (2001), "Passive energy dissipation device for typical steel frame building in Iran", Engineering Structures, Vol. 23, pp. 643-655. DOI: 10.1016/S0141-0296(00)00082-1

Tena, A y H J Nangullasmú (2013), "Diseño sísmico de marcos no dúctiles de concreto reforzado con disipadores de energía histeréticos. Definición de parámetros de diseño", Revista Internacional de Desastres Naturales, Accidentes e Infraestructura Civil, Vol. 13, No. 2, pp. 275-299. ISSN 1936-1483

Tena-Colunga, A, H Correa-Arizmendi, J L Luna-Arroyo y G Gatica-Avilés (2008), "Seismic behavior of code-designed medium rise special moment-resisting frame RC buildings in soft soils of Mexico City”, Engineering Structures, Vol. 30, pp. 3681-3707. DOI: 10.1016/j.engstruct.2008.05.026

Tena-Colunga, A y H J Nangullasmú-Hernández (2015), “Assessment of seismic design parameters of moment resisting RC braced frames with metallic fuses", Engineering Structures, Vol. 95,pp. 138-153. DOI: 10.1016/j.engstruct.2015.03.062 
Vargas, R y M Bruneau (2009), “Analytical response and design of buildings with metallic structural fuses. I”, ASCE Journal of Structural Engineering, Vol. 135, No. 4, pp. 386-393. DOI: 10.1061/(ASCE)0733-9445(2009)135:4(386)

Wallace, J y J P Moehle (1989), "BIAX: A computer program for the analysis reinforced concrete sections", Report No. UCB/SEMM-89/12, Department of Civil Engineering, University of California at Berkeley.

Wu, B, J P Ou y T T Soong (1997), “Optimal placement of energy dissipation devices for three-dimensional structures”, Engineering Structures, Vol. 19, No.2, pp. 113-125. DOI: 10.1016/S0141-0296(96)00034$\underline{X}$

Zahrai, S M y A Moslehi (2013), "Analytical study on cyclic behavior of chevron braced frames with shear panel system considering post-yield deformation", Canadian Journal of Civil Engineering, Vol. 40, pp. 633-643. DOI: $10.1139 /$ cjce-2012-0430 